\title{
Ensayo geometalúrgico para la recuperación de oro de la zona de sulfuros primarios de la mina La Herradura, Sonora, México
}

\author{
Efrén Pérez-Segura, Juan Miguel Romero-Valle, Gabriela Orozco-Rivera, Daniel Rosales-Suárez, \\ Rodrigo Martínez-Peñuñuri
}

Efrén Pérez-Segura

efrenpese@yahoo.com

Juan Miguel Romero-Valle

Departamento de Geología, Universidad de

Sonora. Rosales y Bd. Luis Encinas. 83000

Hermosillo, Sonora, México.

\section{Juan Miguel Romero-Valle}

Gerencia de Proyectos Mineros, Zona Noroeste, Fresnillo, PLC., Caborca, Sonora, México.

\section{Gabriela Orozco-Rivera \\ Daniel Rosales-Suárez}

Centro de Investigación y Desarrollo Tecnológico Servicios Especializados Peñoles S.A. de C.V. Prolongación Comonfort 2050. Antigua Aduana Col. Luis Echeverría, C.P. 27300

Torreón, Coahuila, México.

\section{Rodrigo Martínez Peñuñuri}

Laboratorio Tecnológico de Metalurgia LTM, S.A. de G.V. Carretera a Tecoripa km. 3.5 No. 351. Col. Parque Industrial, 83259, Hermosillo, Sonora, México.

BOL. SOC. GEOL. MEX. 2019

VOL. 71 NO. 1

P. $139-168$

http://dx.doi.org/10.18268/BSGM2019v7 lnla8

Manuscrito recibido: Agosto 28, 201 Manuscrito corregido: Abril 4, 2018. Manuscrito aceptado: Abril 10, 2018

\section{RESUMEN}

La mina La Herradura, en Sonora, constituve uno de los distritos de oro más importantes de México, con una producción a la fecha superior a 5.5 millones de onzas de oro durante 17 años. Forma parte de un cinturón de yacimientos de oro orogénico que se extiende en dirección noroeste-sureste por más de $300 \mathrm{~km}$ de largo y $50 \mathrm{~km}$ de ancho. La mineralización consiste en vetas y enrejados de vetillas de cuarzo formadas en un ambiente geológico dúctil-frágil, que se hospeda en gneises cuarzo feldespáticos del Proterozoico. Los cuerpos mineralizados, definidos por una ley mínima de corte (cut off) de $0.3 \mathrm{~g} / \mathrm{t}$ de Au tienen formas tabulares y alcanzan hasta $1 \mathrm{~km}$ de largo, $1 \mathrm{~km}$ de profundidad y $100 \mathrm{~m}$ de ancho. El control visual de la mineralización es la constancia de vetas y vetilla de cuarzo y una alteración hidrotermal sericítica persistente. Para este trabajo se utilizaron diferentes técnicas, poniendo énfasis en el programa Minera Liberation Analizer (MLA), con el objeto de probar la posibilidad de predecir el oro recuperable en la mina, para la zona de sulfuros primarios. Se definieron 3 zonas geometalúrgicas (Zonas A, B y C) de las cuales se hicieron compósitos y concentrados gravimétricos. La composición modal de los concentrados es de cuarzo, feldespato y muscovita (sericita) y una mineralogía metálica de pirita, esfalerita, galena, magnetita, oro y telururos de oro y plata. El oro se identifica como inclusiones en pirita o en diversas gangas como cuarzo, albita, ortoclasa o ankerita, así como en películas en el borde pirita. La composición del oro es electrum con $74 \%$ de Au y $26 \%$ de Ag; también se identificó la presencia de petzita $\left(\mathrm{Ag}_{3} \mathrm{AuTe} \mathrm{C}_{2}\right)$ y stutzita (AgTe). Se calcularon constantes de recuperación para cada zona geometalúrgica las cuales, incorporadas al modelo informático de recursos calculados en más de 14 millones de onzas de oro, indicaron rangos de error en oro recuperable de menos de $4 \%$ para la Zona A, $6 \%$ para la Zona $\mathrm{C}$ y $13 \%$ para la Zona B, en relación con cálculos tadicionales efectuados con datos experimentales. Los resultados son aceptables para aplicar esta metodología al depósito. El rango de error más acusado en la Zona B se interpreta como causado por un "efecto pepita" muy común en este tipo de yacimientos. Se concluye también que un proceso de remolienda, incorporado actualmente a la planta de beneficio, es probablemente innecesario, lo cual implicaría un ahorro notable en energía y en la economía de la mina.

Palabras clave: La Herradura, oro, geometalurgia, Mineral Liberation Analizer, Sonora, México.

\section{ABSTRACT}

La Herradura mine in Sonora is one of the most important gold districts in Mexico producing more than 5.5 million ounces of gold over 17 years. It is part of an orogenic gold deposits belt with a northwest-southeast direction for $300 \mathrm{~km}$ long and $50 \mathrm{~km}$ wide. The mineralization consists of veins and quartz networks veinlets formed in a brittle-ductil geologic environment and it is hosted in Proterozoic quartz-feldspar gneisses. The ore bodies, defined by a $0.3 \mathrm{~g} / \mathrm{t}$ Au cut-off grade, have tabular forms up to $1 \mathrm{~km}$ in length, $1 \mathrm{~km}$ in depth and $100 \mathrm{~m}$ in width. The visual control for mineralization is the abundance of quartz veins and veinlets, and a persistent sericitic hydrothermal alteration. Different techniques were used in this work with special focus on the Mineral Liberation Analizer (MLA) program to prove the possibility of predicting recoverable gold in the mine for the primary sulphide zone. Three geometallurgical zones (Zones $A, B$ and $C$ ) were defined by ore composites and gravimetric concentrates from the same composites. Modal composition of the concentrates is quartz, feldspar and muscovite (sericite), and a metallic mineralogy of pyrite, sphalerite, galena, magnetite, gold and tellurides of gold and silver. Gold is identified as inclusions in pyrite or in gangue minerals like quartz, albite, orthoclase or ankerite, as well as coating pyrite crystals. The gold composition is electrum with $74 \%$ $A u$ and $26 \% \mathrm{Ag}$; the presence of petzite $\left(\mathrm{Ag}_{3} \mathrm{AuT \textrm {T } _ { 2 }}\right)$ and stutzite $(\mathrm{AgT}$ ) ) were also identified. Recovery constants were calculated for each geometallurgical zone, which were introduced to the resource model of more than 14 million ounces of gold, indicating that error range in recoverable gold is less than 4\% for Zone $A$, $6 \%$ for Zone C and 13\% for Zone B, in relation with gold recovery calculated with traditional methods. These results could be acceptable to applicate this methodology to La Herradura deposit. The most important error range in the Zone $B$ is interpreted as due to a nugget effect, which is very common in such mineral deposits. It is also concluded that the secondary milling process currently incorporated to the metallurgical plant is probably unnecessary, so its removal would result in a significant saving in energy and therefore in the economy of the mine.

Keyzerds: La Herradura, gold, geometallurgy, Mineral Liberation Analizer, Sonora, Mexico. 


\section{Introducción}

Las bondades de la mineralogía aplicada a los procesos de explotación y beneficio de los minerales de mena es bien conocida desde hace mucho tiempo (Amstutz, 1961; Hagni, 1978; Petruk, 2000; Pérez-Segura, 2003; Lamberg, 2011). En los últimos 20 años ha emergido la geometalurgia como una disciplina que integra múltiples aspectos relacionados con la geología económica y con la economía de minas y proyectos mineros (Dobby et al., 2004; Hallewell, 2009; Keeney, 2010; Birtel et al., 2011; Bye, 2011; Lamberg, 2011; Pérez-Segura et al., 2013). La geometalurgia basa su principio en la comprensión de que las menas son asociaciones de minerales, no de elementos químicos y, puesto que todos los procesos metalúrgicos tratan con minerales, son las características fisicoquímicas de éstos las que determinan las condiciones de un proceso de recuperación industrial (Lamberg, 2011). Algunas de las bases que sustenta la geometalurgia se enlistan en el Anexo 1 (Pérez-Segura et al., 2013).

De lo anterior se deduce que un modelo geometalurgico es más avanzado que el modelo geológico de yacimiento en una mina en producción, ya que debería ser capaz de predecir la recuperación real del metal de interés desde antes de la explotación (Dobby et al., 2004).

La técnica básica para el estudio de los minerales de mena y sus texturas es la mineralografía o microscopía de reflexión, descrita ampliamente en la gran obra de Ramdohr (1980). Esta técnica es realmente la mejor para el estudio de minerales metálicos en relación costo/beneficio; su debilidad es que se trata de un método cualitativo y puntual, no obstante los esfuerzos desarrollados para realizar cuantificaciones sistemáticas (Pérez-Barnuevo et al., 2009). Son las técnicas de microscopía electrónica de barrido con energía dispersiva (MEB-EDS) las que han permitido el desarrollo de los sistemas de identificación mineralógica y cuantificación de partículas más eficaces desde hace 40 años (Hall, 1977). Este desarrollo ha desembocado en dos sistemas analíticos que dominan actualmente el mercado y que se conocen como
Quantitative Evaluation of Minerals by SCANning electron microscopy (QEM-SCAN) (Miller et al., 1982; Reid et al., 1984; Gottlieb et al., 2000; Goodal y Scales, 2007; Hoal et al., 2009) y el Mineral Liberation Analizer (MLA) (Gu, 2003; Goodall y Scales, 2007; Fandrich et al., 2007; Hunt et al., 2011; Hartner, 2012) y, recientemente, se habla también de un sistema nuevo llamado Advanced Mineral Identification and Characterization System (AMICS) (Zhou y Gu, 2016). No existe en la literatura gran información sobre aplicaciones de la geometalurgia a yacimientos de oro, muy probablemente debido a que un problema fundamental es el muestreo de este tipo de yacimientos (Pitard, 1989a y b; Goodall y Scales, 2007). Los trabajos más destacados con sistemas auríferos proceden de tesis desarrolladas en universidades australianas (Leichliter et al., 2011; Bonnici, 2012; Leichliter, 2013).

El objeto del presente trabajo es el de dar a conocer los resultados más relevantes de un ensayo de modelo de recuperación geometalúrgica efectuado en la mina La Herradura en Sonora (Romero-Valle, 2017), una de las minas de oro más importantes de México, tomando en cuenta el análisis de la información obtenida a partir de estudios con microscopía de reflexión, concentración gravimétrica, Mineral Liberation Analyzer (MLA) y pruebas de cianuración dinámica. Todos estos elementos, incorporados al modelo de cálculo de recursos, permiten demostrar que la geometalurgia es una herramienta multidisciplinaria con un gran porvenir en el desarrollo minero del país.

\section{Geología y yacimientos minerales de la mina La Herradura}

Una provincia de yacimientos de oro orogénico en el sentido descrito por varios autores (Groves et al., 1998; Goldfarb et al., 2001, 2008), ha sido definida desde hace casi más de 30 años en Sonora (Pérez-Segura et al., 1989; Albinson, 1989; Ochoa-Landín et al., 2011). Este tipo de depósitos (Figura 1) se identifica sobre un cinturón de rocas deformadas en la provincia del Desierto 
de Sonora conocido como Mojave-Sonora Megashear (Anderson y Silver, 1979). El cinturón incluye minas y yacimientos como San Francisco (Pérez-Segura et al., 1989), El Chanate (Silberman et al., 1988), Tajitos (Meza-Figueroa, 1992; Noriega-Martínez, 2006), La Choya (Durgin y Terán, 1996; Thoms, 1998), Sierra Pinta (Araux-Sánchez, 2000), Karina (Ornelas-Macías y Verdugo-Noriega, 2009), Campo Juárez (Pérez-Segura et al., 1989), Soledad-Dipolos, Nochebuena y La Herradura (Romero-Valle, 2005, 2017; Quintanar-Ruiz, 2008; de la Torre, 2009, 2010), además de otros compilados por Izaguirre et al. (2012, 2017).

El distrito minero La Herradura es la más importante de las zonas mineralizadas y se extiende sobre una superficie de 15 por $15 \mathrm{~km}$ (Figura 2). Los depósitos están hospedados en un bloque del basamento Proterozoico limitado por fallas tanto al Este (Falla Victoria) como al Oeste (Falla Ocotillo). El bloque del Este se compone de rocas metavolcánicas y metasedimentarias del Jurásico (Quintanar-Ruiz, 2008; Izaguirre-Pompa et al., 2009, 2012); el del Oeste está constituido por calizas, dolomías y cuarcitas del Paleozoico (Poole et al., 2004). Por otra parte, rocas intrusivas del Cretácico afloran en el Cerro Los Indios (Izaguirre et al., 2012), al Suroeste del distrito y gruesos depósitos de arena cubren la región Oeste.

El basamento Proterozoico que aloja la mineralización de oro de La Herradura está compuesto por

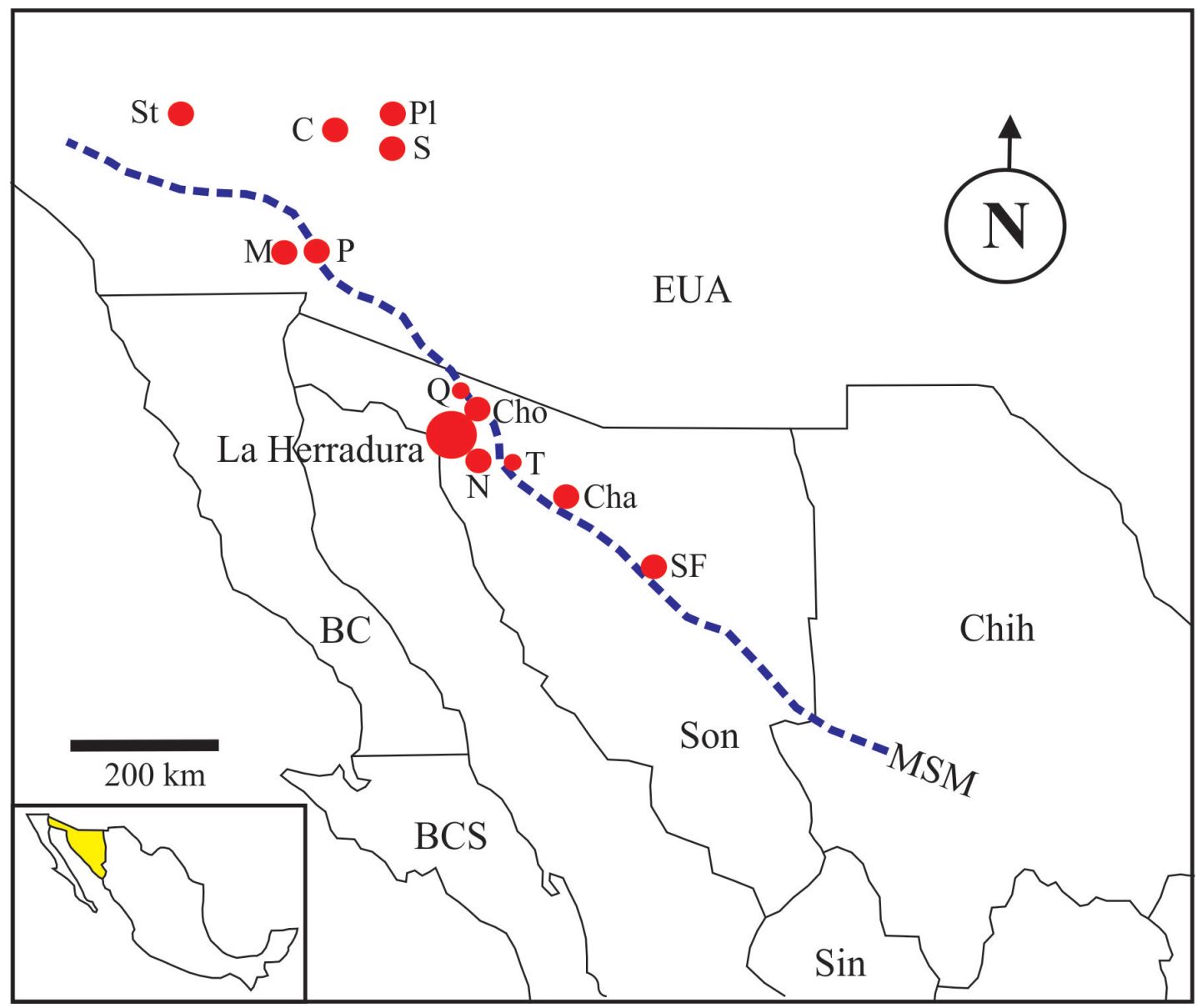

Figura 1 Mapa de localización de algunos yacimientos de oro orogénico en Sonora a lo largo del Mojave-Sonora Megashear (Anderson y Silver, 1979). Se incluyen algunos yacimientos en Estados Unidos según Titley y Zürcher en Romero-Valle (2017). SF-San Francisco, Cha-El Chanate, T-Tajitos, N-Nochebuena, Cho-La Choya, Q-Quitovac, M-Mezquite, P-Picacho, St-Steadman, C-Copperstone, PI-Planet, S-Swansen. MSM-Mojave-Sonora Megashear. 


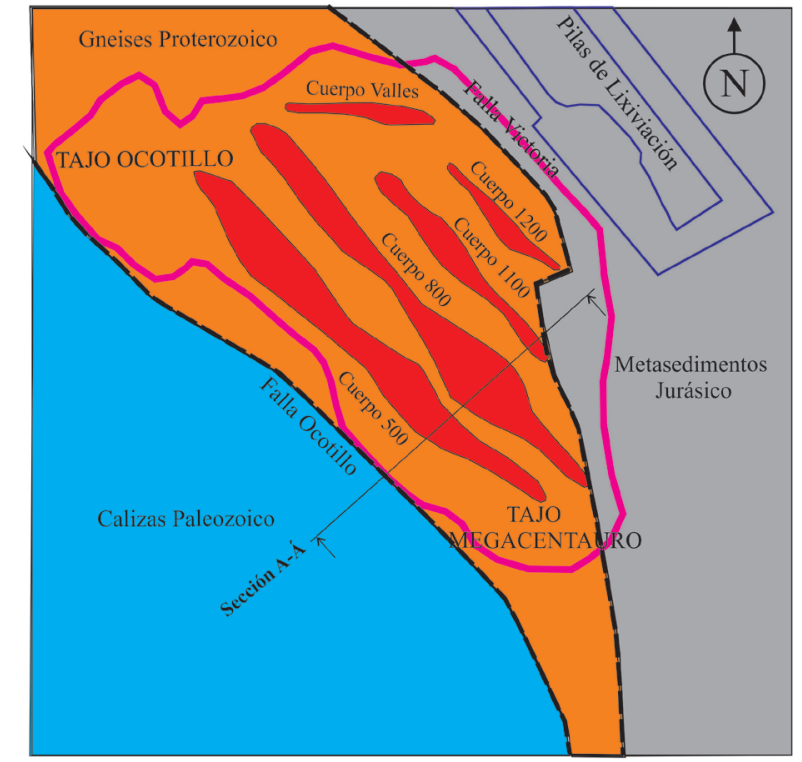

A

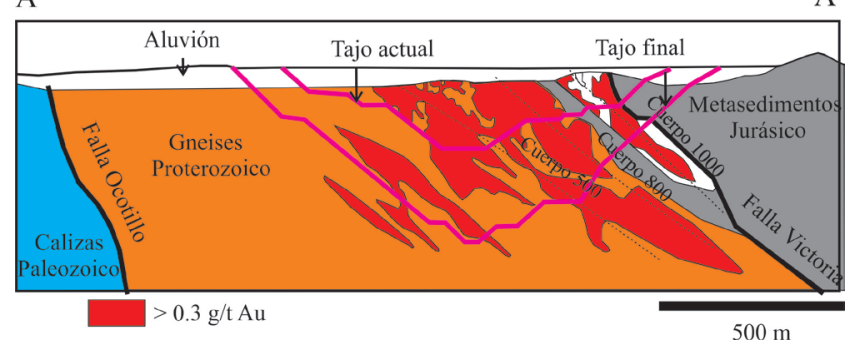

Figura 2 Mapa geológico simplificado y sección en la mina La Herradura, mostrando los principales cuerpos minerales (de la Torre, 2010, modificado).

gneises en grado de anfibolita fechados entre 1.75 y 1.65 Ga por U-Pb en zircones (Quintanar-Ruiz, 2008; Izaguirre et al., 2017). La edad más relacionada con la mineralización de oro es de $61 \mathrm{Ma}$ (Quintanar-Ruiz, 2008) obtenida por Re-Os en pirita y magnetita. Esta edad se aproxima a las interpretadas para la mineralización de oro en la región por Iriondo et al. (2005), entre 65 y 48 Ma en base a fechamientos por ${ }^{40} \mathrm{Ar} /{ }^{39} \mathrm{Ar}$ usando muscovita de las zonas de cizalla.

El distrito de oro La Herradura se localiza a lo largo de un sistema de cizalla con una tendencia favorable Noroeste-Sureste. El sistema está bordeado por las fallas Victoria y Ocotillo y contiene los cuerpos de mineral en los tajos MegaCentauro y Valles (Figura 2). Las fallas delimitan el cinturón metamórfico proterozoico que se compone principalmente de gneises de biotita, gneises cuarzo-feldespáticos, pegmatitas y esquistos verdes y marrones. El gneis cuarzo-feldespático es la roca más favorable para el depósito de minerales de oro. La favorabilidad para el depósito se interpreta como relacionada con el comportamiento reológico de la roca.

\subsection{GARACTERÍSTICAS GENERALES DE LOS GUERPOS DE MINERAL}

En el distrito La Herradura se han obtenido, durante los 17 años de operación de la mina, 5.4 millones de onzas de oro. Los cuerpos de mineral de La Herradura consisten en conjuntos de vetas de cuarzo desarrolladas en un ambiente tectónico frágil-dúctil (Figura 3). La mayoría de las vetas están alojadas en gneises cuarzo-feldespáticos de muscovita. Sin embargo, algunas vetas formales pueden penetrar hasta varios metros en los gneises de biotita y por unos pocos centímetros en las rocas meta-sedimentarias del Jurásico. Los espesores de las vetas individuales varían desde unos pocos centímetros hasta 5 metros y sus longitudes varían de los $3 \mathrm{~m}$ hasta $100 \mathrm{~m}$. Las vetas se pueden asociar también en redes complejas (Figura 3).

La tendencia de las vetas de cuarzo aurífero es al Noroeste y generalmente están inclinadas hacia el Noreste. También presentan arreglos de vetillas en echelón al alto y al bajo de las estructuras mineralizadas principales. De acuerdo con la geometría, las vetas de cuarzo aurífero pueden desarrollarse en tres tipos de estructuras: a lo largo de fallas de cabalgamiento, en fallas extensionales en echelón y en fallas conjugadas (Figura 3).

Los cuerpos de mineral económico en La Herradura (Figura 2) tienen una forma tabular y pueden alcanzar una dimensión de $1 \mathrm{~km}$ de largo (tendencia Noroeste y echado al Noreste), $1 \mathrm{~km}$ de profundidad y $100 \mathrm{~m}$ de ancho (vista en planta). En general, estos yacimientos se definen económicamente por una ley mínima de corte (cut-off) de $0.30 \mathrm{~g} / \mathrm{t}$ de $\mathrm{Au}$. El tamaño medio de los cuerpos de mineral económico basado en esta ley de corte alcanza anchos de $10 \mathrm{~m}$ a $100 \mathrm{~m}$ y longitudes de 500 a 700 m en planta. El control visual característico de la ley para estos cuerpos de mineral 

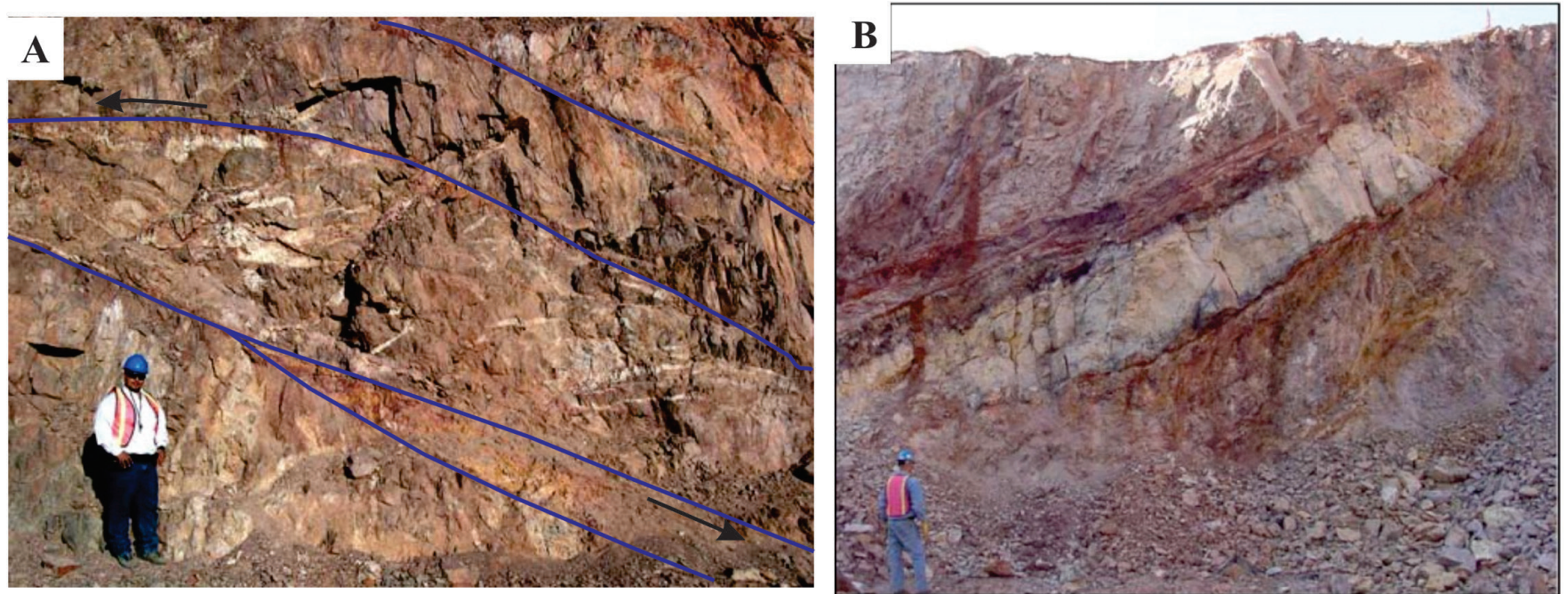

Figura 3 Estilo de la mineralización en la mina La Herradura. A: zona de cizalla en fallamiento inverso con vetas y vetillas de cuarzo deformadas. B: Veta de cuarzo en tajo Megacentauro, banco 16, con una ley superior a $15 \mathrm{~g} / \mathrm{t} \mathrm{Au}$.

económico es la abundancia de vetas observadas. Otra guía para la mineralización es la alteración sericítica, la cual es persistente a escala kilométrica en las zonas mineralizadas.

Las vetas se componen principalmente de cuarzo con menor pirita, esfalerita, galena y oro nativo. Los sulfuros representan menos de 10 por ciento del volumen de las vetas y carecen de una zonificación vertical o lateral con respecto a las leyes de oro. La mayoría de las vetas mineralizadas contienen una ley promedio superior a $5 \mathrm{~g} / \mathrm{t}$ de $\mathrm{Au}$, pero algunas muestras pueden alcanzar leyes superiores a 100 $\mathrm{g} / \mathrm{t}$ de $\mathrm{Au}$. En estos casos es común la presencia de oro visible. Además de cuarzo, la mineralogía común de las vetas es pirita, carbonato ferrífero y clorita. Los minerales de alteración hidrotermal más comunes en la roca encajonante son sericita y, en menor proporción, carbonato y pirita.

\section{Ensayo geometalúrgico para la recuperación de oro en la mina La Herradura}

\subsection{DEFINIGIÓN DE LAS ZONAS PARA SU ESTUDIO}

Puesto que las características de la mineralización a la escala del afloramiento y dado que la litología y la alteración hidrotermal en la zona de estudio son homogéneas, la selección de zonas geometalúrgicas se hizo a partir de la constancia de sulfuros primarios, los cuales se identifican a partir del banco -96 (metros por debajo del nivel del mar). Dos de las zonas son exclusivamente de sulfuros primarios y una de mezcla sulfuros-óxidos. Las zonas corresponden a la exploración profunda realizada en los tajos MegaCentauro, Ocotillo y Valles, dentro de los cuales está la mayor parte de recursos estimados para la mina.

Una problemática característica para este tipo de yacimientos es la presencia de oro grueso, lo cual hace complejo el muestreo representativo debido a un "efecto pepita" muy acusado ya reconocido anteriormente en La Herradura (Cossio et al., 2003). El "efecto pepita" extiende su problema al minado y beneficio de este tipo de depósitos. La experiencia en el muestreo y análisis de barrenos de producción ha permitido minimizar desviaciones en la ley de oro analizada en la zona de óxidos. Sin embargo, cuando la mina alcance en su totalidad la zona de sulfuros primarios, al problema analítico del oro se adicionará el problema de la recuperación metalúrgica.

Para seleccionar las zonas se hizo la descripción geológica de cada una de las áreas con base en la cartografía del tajo y a observaciones en núcleos 
de barrenos de diamante. Se definieron los barrenos a utilizar con base en litología, alteración hidrotermal, mineralogía y leyes de oro, con lo cual se seleccionaron las zonas llamadas A, B y C (Figura 4). La descripción litológica de los tramos que intervinieron en los compósitos de cada una de las áreas se presenta en la Tabla 1.

\subsection{TÉGNICAS Y MÉTODOS EMPLEADOS}

El solo proceso de preparación de los compósitos representativos fue arduo (un mes de trabajo). Todas las preparaciones se hicieron en las instalaciones de la unidad minera La Herradura. La metodología de elaboración de los compósitos de mineral y del trabajo posterior a partir de los mismos se da en el Anexo 2.
Se efectuaron secciones pulidas de muestras de núcleo seleccionadas, así como de los concentrados gravimétricos obtenidos a partir de los compósitos. Las secciones pulidas fueron efectuadas en el laboratorio del Departamento de Geología de la Universidad de Sonora y en los laboratorios del Centro de Investigación y Desarrollo Tecnológico de Peñoles en Torreón, Coahuila. La metodología de manufactura fue de pulido al alto brillo utilizando abrasivos y emulsiones de diamante de diferente tamaño de grano. El estudio mineralográfico se hizo con microscopios Leitz-Orthoplan y Nikon-Labophot T2-POL.

La concentración gravimétrica del material de los compósitos se efectuó con un concentrador gravimétrico Knelson KC-MD3 propiedad del Laboratorio Tecnológico de Metalurgia en Hermosillo,

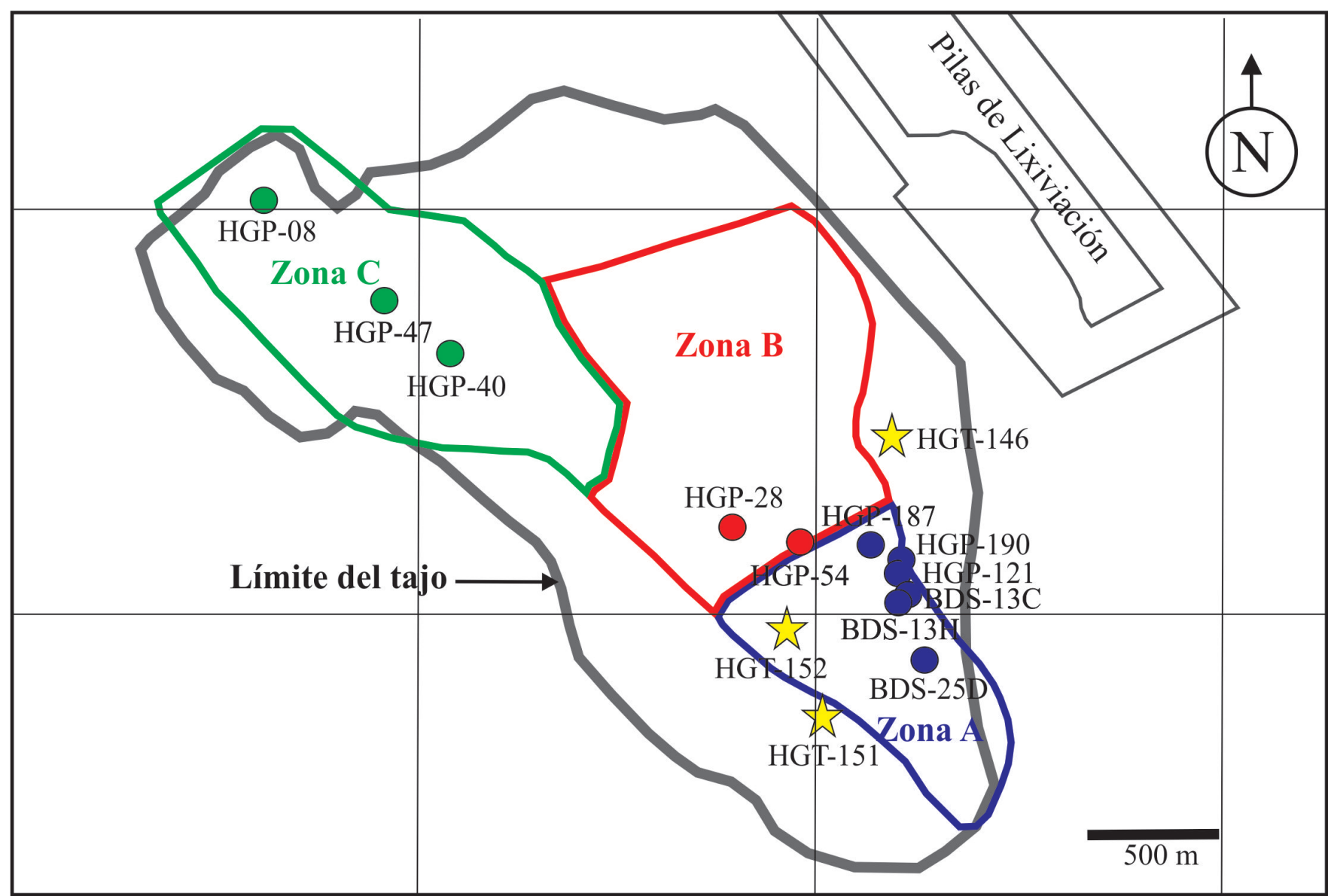

Figura 4 Definición de las zonas geometalúrgicas A, B y C. Círculos: barrenos utilizados para los compósitos de concentración gravimétrica; estrellas: barrenos muestreados para estudiar el oro in situ. 
Tabla 1. Características geológicas de los barrenos y tramos de las zonas muestreadas.

\begin{tabular}{|c|c|c|}
\hline Barreno & Profundidad & Zona \\
\hline$B D S-13-C$ & $592.20-614.10$ & A-Sulfuros \\
\hline BDS-13-H & $552.30-583.20$ & A-Sulfuros \\
\hline$B D S-25-D$ & $354.90-360.55$ & A-Óxidos \\
\hline$H P G-121$ & $633.30-648.50$ & A-Sulfuros \\
\hline$H P G-121$ & $682.25-699.60$ & A-Sulfuros \\
\hline$H P G-187$ & $446.20-463.55$ & A-Sulfuros \\
\hline$H P G-190$ & $642.55-668.55$ & A-Sulfuros \\
\hline$H P G-28$ & $112.85-198.85$ & B-Óxidos-Sulfuros \\
\hline$H P G-54$ & $166.95-185.55$ & B-Óxidos-Sulfuros \\
\hline$H P G-08$ & $644.65-668.40$ & C-Sulfuros \\
\hline
\end{tabular}

Sonora. El material utilizado provino de los compósitos representativos de las zonas $\mathrm{A}, \mathrm{B}$ y $\mathrm{C}$ molidos a un tamaño de $-0.841 \mathrm{~mm}$ (-20 mallas); para cada prueba se utilizaron $5 \mathrm{~kg}$ de material. El concentrador fue diseñado y balanceado dinámicamente para operar a $1500 \mathrm{rpm}$ que corresponde a una velocidad de aceleración de 60 G's, con un flujo de alimentación de 2 galones de agua por minuto y una presión de fluidización de 7 PSI; pero también se hicieron pruebas a 90 G's y 120 G's para buscar la mejor recuperación durante la concentración.

Los estudios de caracterización mineralógica fueron realizados mediante la técnica de análisis modal con el sistema de Mineral Liberation Analyzer (MLA), el cual consiste en un programa informático de análisis de partículas muy robusto, adaptado a un microscopio electrónico de barrido con espectrometría de dispersión de energía de rayos $\mathrm{X}$ (MEB-EDS). La técnica consiste en el análisis de los rayos $\mathrm{X}$ producidos por la muestra al ser excitada por una fuente de electrones. Para la identificación mineralógica se utilizaron las imágenes de electrones retrodispersados. La técnica permite automatizar la extracción de la información sobre la asociación y composición química de las partículas minerales generando los datos estadísticos más significativos para un análisis modal y textural de las partículas. El equipo utilizado fue un microscopio electrónico de barrido marca FEI, modelo Quanta 600, propiedad del Centro de Investigación y Desarrollo Tecnológico de Peñoles en Torreón, Coahuila. Las mediciones para la mineralogía se hicieron en modo XBSE (modo de análisis donde los granos minerales son discriminados por las imágenes de electrones retrodispersados) y en modo Sparse Phase Liberation (SPL - liberación de fases dispersas) para la búsqueda del oro. Las condiciones de operación fueron a un voltaje de $25 \mathrm{KV}$, un spot-size de 7.07.2 , distancia de trabajo de $10 \mathrm{~mm}$, contraste de 45 y brillo de 75 .

Las pruebas metalúrgicas de cianuración dinámica en botellas se efectuaron sobre muestras de testigos de los compósitos de las tres zonas en los laboratorios metalúrgicos de la Unidad Minera La Herradura de la compañía Fresnillo, PLC. Las condiciones fueron de 1000 ppm de NaCN, 
$3 \mathrm{~kg} /$ ton de $\mathrm{CaO}, 40 \%$ de sólidos y un tiempo de 96 horas. Una prueba se hizo a un tamaño de partícula de -20 mallas y otra a -200 mallas.

Los cálculos de oro recuperado se hicieron en el Departamento de Geología de la mina La Herradura, para lo cual se utilizó el software TSS Studio propiedad de Newmont, mismo que se utiliza en el modelo geológico para calcular los recursos y reservas del yacimiento.

\subsection{RESULTADOS OBTENIDOS}

\subsubsection{CONGENTRADOS GRAVIMÉTRICOS}

El objeto de efectuar las concentraciones gravimétricas fue doble:
- Concentrar aún más el oro existente para facilitar el estudio.

- Comparar resultados con los que se obtienen a escala industrial en el proceso metalúrgico actual, tomando en cuenta que en el proceso metalúrgico vigente existen incorporados concentradores gravimétricos antes de la lixiviación dinámica.

Las concentraciones gravimétricas se realizaron a las aceleraciones de 60, 90 y 120 G's.

Las leyes de cabeza de los compósitos analizadas al fuego fueron, para la Zona A: $9.79 \mathrm{~g} / \mathrm{t}$ Au y 17 g/t Ag, para la Zona B: 2.52 g/t Au y <5 g/t Ag y para la Zona C: $1.34 \mathrm{~g} / \mathrm{t} \mathrm{Au} \mathrm{y}<5 \mathrm{~g} / \mathrm{t} \mathrm{Ag}$.

La Tabla 2 y la Figura 5 muestran los balances metalúrgicos para los mejores resultados de

Tabla 2. Pruebas de concentración gravimétrica con los mejores resultados de recuperación de oro.

\section{Mejores pruebas de concentración gravimétrica}

Zona A PK2 a (90 G's)

\begin{tabular}{|c|c|c|c|c|c|c|c|c|c|}
\hline \multirow[t]{2}{*}{ Producto } & \multirow{2}{*}{$\begin{array}{l}\text { Peso } \\
\text { (Kgs) }\end{array}$} & \multirow{2}{*}{$\begin{array}{c}\text { Peso } \\
\text { \% }\end{array}$} & \multicolumn{2}{|c|}{ Análisis (ppm) } & \multicolumn{2}{|c|}{ Contenido (mg) } & \multicolumn{2}{|c|}{$\begin{array}{c}\text { Recuperación } \\
\%\end{array}$} & \multirow{2}{*}{$\begin{array}{c}\text { Relación de } \\
\text { Concentración } \\
\text { Au-Ag }\end{array}$} \\
\hline & & & Au & Ag & Au & Ag & Au & Ag & \\
\hline Concentrado & 0.403 & 8.08 & 111.21 & 123 & 44.82 & 49.57 & 78.1 & 51.94 & 12.38 \\
\hline Colas & 4.587 & 91.92 & 2.74 & 10 & 12.57 & 45.87 & 21.9 & 48.1 & \\
\hline Total & 4.99 & 100 & & & 57.39 & 95.44 & 100 & 100.04 & \\
\hline Cabeza Ensayada & & & 9.79 & 17 & & & & & \\
\hline Cabeza Calculada & & & 11.5 & 19.13 & & & & & \\
\hline C.F & & & 1.17 & 1.13 & & & & & \\
\hline \multicolumn{10}{|l|}{ Zona B PK1 a (90 G's) } \\
\hline \multirow[t]{2}{*}{ Producto } & Peso & Peso & \multicolumn{2}{|c|}{ Análisis (ppm) } & \multicolumn{2}{|c|}{ Contenido (mg) } & \multicolumn{2}{|c|}{$\begin{array}{c}\text { Recuperación } \\
\%\end{array}$} & $\begin{array}{c}\text { Relación de } \\
\text { Concentración }\end{array}$ \\
\hline & (Kgs) & $\%$ & Au & Ag & Au & Ag & Au & Ag & Au-Ag \\
\hline Concentrado & 0.163 & 3.26 & 79.66 & 55 & 12.98 & 8.96 & 83.79 & 100 & 30.64 \\
\hline Zona C PK1 a (60 G's) & 4.832 & 96.74 & 0.52 & 0 & 2.51 & 0 & 16.21 & 0 & \\
\hline Total & 5 & 100 & & & 15.5 & 8.96 & 100 & 100 & \\
\hline Cabeza Ensayada & & & 2.52 & 2 & & & & & \\
\hline Cabeza Calculada & & & 3.1 & 1.79 & & & & & \\
\hline C.F & & & 1.23 & 0.9 & & & & & \\
\hline \multicolumn{10}{|l|}{ Zona C PK1 a (60 G's) } \\
\hline \multirow[t]{2}{*}{ Producto } & Peso & Peso & \multicolumn{2}{|c|}{ Análisis (ppm) } & \multicolumn{2}{|c|}{ Contenido (mg) } & \multicolumn{2}{|c|}{$\begin{array}{c}\text { Recuperación } \\
\%\end{array}$} & $\begin{array}{c}\text { Relación de } \\
\text { Concentración }\end{array}$ \\
\hline & (Kgs) & $\%$ & Au & Ag & Au & Ag & Au & Ag & Au-Ag \\
\hline Concentrado & 0.176 & 3.77 & 75.76 & 60 & 13.33 & 10.56 & 74.6 & 70.15 & 26.53 \\
\hline Colas & 4.494 & 96.23 & 1.01 & 1 & 4.54 & 4.49 & 25.4 & 29.95 & \\
\hline Total & 4.67 & 100 & & & 17.87 & 15.05 & 100 & 100 & \\
\hline Cabeza Ensayada & & & 1.34 & 3 & & & & & \\
\hline Cabeza Calculada & & & 3.83 & 3.22 & & & & & \\
\hline C.F & & & 2.86 & 1.07 & & & & & \\
\hline
\end{tabular}


recuperación obtenidos en cada una de las pruebas. En la tabla se muestra las leyes de cabeza analizadas y las leyes calculadas en base al balance metalúrgico. A continuación, se describen las características más importantes de los concentrados obtenidos:

\section{Zona A:}

La mejor recuperación para oro fue de $78.10 \%$ a una aceleración de 90 G's. La ley de oro del concentrado fue de $111.21 \mathrm{~g} / \mathrm{t}$ de $\mathrm{Au}$, la ley de cola de $2.74 \mathrm{~g} / \mathrm{t} \mathrm{Au}$, una ley de cabeza ensayada de $9.79 \mathrm{~g} / \mathrm{t} \mathrm{Au}$, una ley de cabeza calculada de 11.5 $\mathrm{g} / \mathrm{t}$ Au y una relación de concentración de 12.38 $\mathrm{kg}$ de concentrado por tonelada de mineral. La recuperación para las otras pruebas fue de $44 \%$ a 60 G's y de $74 \%$ a 120 G's.

\section{Zona B:}

Para la zona B la mejor recuperación fue de $84 \%$ a una aceleración de 90 G's. La ley de oro de concentrado fue de $79.7 \mathrm{~g} / \mathrm{t} \mathrm{Au}$, la ley de cola de 0.5 $\mathrm{g} / \mathrm{t} \mathrm{Au}$, una ley de cabeza ensayada de $2.5 \mathrm{~g} / \mathrm{t} \mathrm{Au}$, una ley de cabeza calculada de $3.1 \mathrm{~g} / \mathrm{t}$ Au y una relación de concentración de $30.6 \mathrm{~kg}$ de concentrado por tonelada de mineral. La recuperación para las otras pruebas fue de $71 \%$ para 60 G's y de $81.9 \%$ para 120 G's.

\section{Zona C:}

En este caso la mejor prueba fue a 60 G's con una recuperación de $74.6 \%$. La ley de oro del concentrado fue $75.8 \mathrm{~g} / \mathrm{t}$ de $\mathrm{Au}$, la ley de cola de $1 \mathrm{~g} / \mathrm{t}$ $\mathrm{Au}$, una ley de cabeza ensayada de $1.3 \mathrm{~g} / \mathrm{t} \mathrm{Au}$, una ley de cabeza calculada de $3.8 \mathrm{~g} / \mathrm{t}$ Au y una relación de concentración de $26.5 \mathrm{~kg}$ de concentrado por tonelada de mineral. La recuperación para las otras pruebas fue de $61.7 \%$ a 90 G's y de $57.8 \%$ a 120 G's.

\subsubsection{ANÁLISIS DE CONCENTRADOS POR MLA}

En total se analizaron 9 briquetas procedentes de los concentrados de las 3 pruebas efectuadas para cada zona metalúrgica.

La Tabla 3 enlista los minerales identificados en porcentaje en peso. Los concentrados se componen principalmente de cuarzo, pirita, feldespato y muscovita (sericita), como se muestra en la Figura 6. El cuarzo es el mineral más abundante en zonas A y B (casi 50\%), mientras que en la zona $\mathrm{C}$ es la mitad; por otra parte, la albita aumenta progresivamente de las Zona A a la C. La ankerita y los óxidos de Fe son típicamente más abundantes en la Zona C. La concentración de pirita en zonas A y B es superior a 20\%, mientras que en la zona $\mathrm{C}$ es casi la mitad de las anteriores. Esto sólo indica

\section{$\%$ de recuperación de oro}

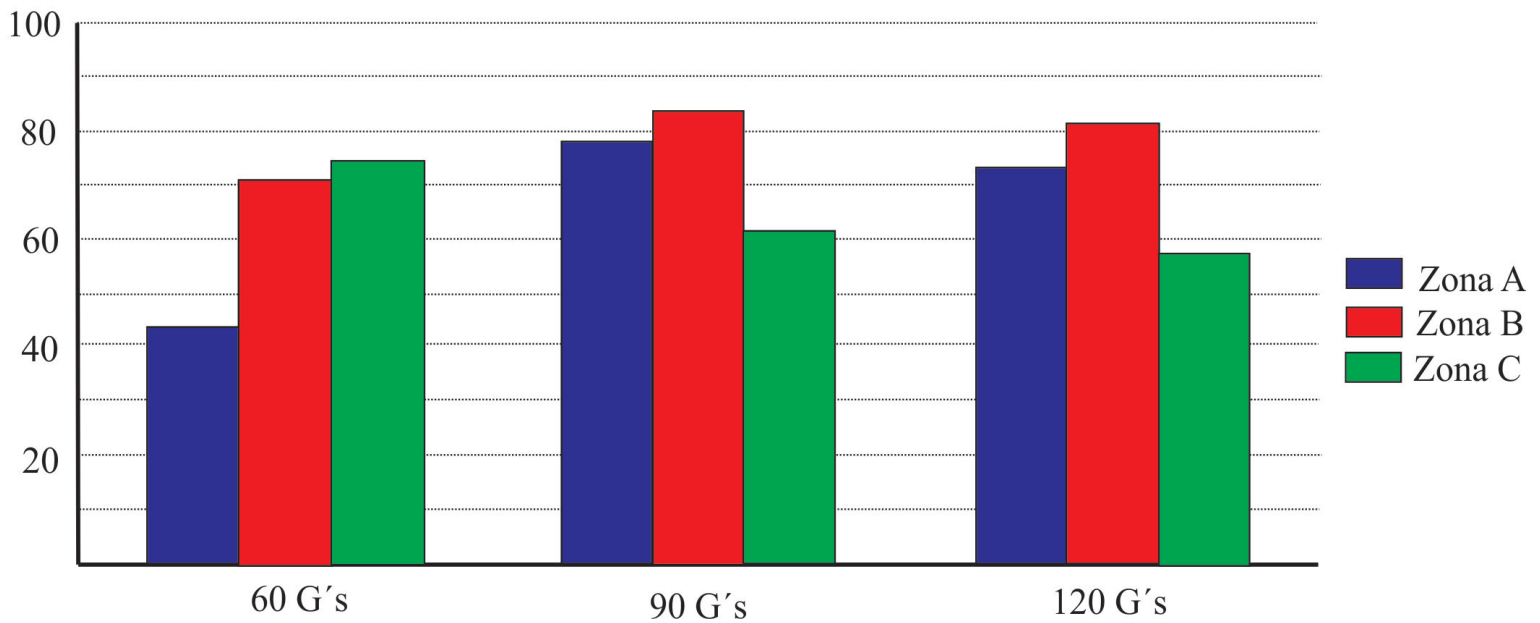

Recuperación de oro con concentración gravimétrica para las zonas A, B y C a aceleraciones de 60,90 y 120 G’s. 
la pobreza de pirita en la zona $\mathrm{C}$ y la concentración de pirita en la zona $\mathrm{B}$, a pesar de ser una zona mixta de óxidos-sulfuros. En pocas proporciones es notable la presencia de galena y esfalerita en la Zona A y una casi ausencia en la Zona C.

Vale la pena insistir en que los porcentajes mineralógicos de los concentrados sólo representan proporciones generales entre minerales más ligeros y más pesados en las muestras generales. La mineralogía es congruente con las características observadas en la litología de los barrenos (Tabla $1)$.

La composición en óxidos mayores para los compósitos de las 3 zonas se puede ver en la Tabla 4 y Figura 6. La geoquímica en óxidos mayores de los compósitos de las zonas A y B son bastante parecidas, y a primera vista son diferentes a la composición de la Zona C. En la Zona C se aprecia un déficit notable de casi $20 \%$ en la cantidad de $\mathrm{SiO}_{2}$ en relación con el contenido en las otras zonas. También la misma Zona $\mathrm{C}$ se caracteriza por una abundancia en $\mathrm{Fe}_{2} \mathrm{O}_{3}, \mathrm{CaO}, \mathrm{MgO}$ y $\mathrm{Na}_{2} \mathrm{O}$ en relación con las zonas A y $\mathrm{B}$.

Tabla 3. Composición mineralógica promedio en porcentaje en peso identificada con Mineral Liberation Analizer (MLA) para los concentrados de las zonas A, B y C.

\begin{tabular}{|l|c|c|c|}
\hline Galena & $\begin{array}{c}\text { ZA Promedio } \\
\text { (3 pruebas) }\end{array}$ & $\begin{array}{c}\text { ZB Promedio } \\
\text { (3 pruebas) }\end{array}$ & $\begin{array}{c}\text { ZC Promedio } \\
\text { (3 pruebas) }\end{array}$ \\
\hline Esfalerita & 2.7 & 0.33 & $s$ \\
\hline Calcopirita & 1 & 0.11 & $<0.01$ \\
\hline Pirita & $<0.01$ & $<0.01$ & 0.02 \\
\hline Cuarzo & 27.56 & 21.84 & 12.24 \\
\hline Muscovita & 47.96 & 46.71 & 22.53 \\
\hline Albita & 8.96 & 8.55 & 9.27 \\
\hline Ankerita & 4.95 & 13.74 & 24.71 \\
\hline Ortoclasa & 3.15 & 2.2 & 12.96 \\
\hline Calcita & 1.45 & 2.64 & 4.55 \\
\hline Clorita & 0.3 & 0.4 & 0 \\
\hline Ox_Fe & 0.43 & 0.09 & 1.72 \\
\hline Andradita & 0.47 & 2.27 & 9.48 \\
\hline Augita & 0.14 & 0.07 & 0.14 \\
Rutilo & 0.24 & 0.25 & 0.58 \\
\hline Apatito & 0.12 & 0.11 & 0.57 \\
Barita & 0.09 & 0.05 & 0.45 \\
\hline Otros & 0 & 0 & 0.13 \\
\hline TOTAL & 0.49 & 0.64 & 0.61 \\
\hline & 100 & 100 & 100 \\
\hline
\end{tabular}

Tomando en cuenta que la presencia de oro está controlada por vetas y vetillas de cuarzo y que existen algunas variaciones en la litología de la Zona $\mathrm{C}$, es claro que la composición en óxidos mayores de los compósitos se puede correlacionar con la relativa abundancia de vetas de cuarzo, así como por la litología de roca, referidas por una mayor o menor presencia de cuarzo libre.

\subsubsection{CARACTERÍSTICAS DEL ORO IDENTIFICADO IN-SITU}

Se estudiaron al microscopio mineralográfico varias secciones pulidas hechas a partir de muestras seleccionadas de 2 barrenos de la Zona A y de uno de la Zona B. No pudieron estudiarse muestras de la Zona C. Para la Zona A fueron las muestras de los barrenos HGT-153@1170.80 m. y HGT-151@1316.25 m.; mientras que para la Zona B se estudió la muestra HGT-146@970.20 m. (figuras 7, 8 y 9). Por la profundidad de las muestras se puede tener una idea de la extensión de la mineralización hasta cerca de mil metros de la superficie actual.

Para las muestras de los barrenos de la Zona A se reconocieron 93 partículas de oro. La media de los largos de las partículas es de $60 \mu \mathrm{m}$ y la media de los anchos es de $40 \mu \mathrm{m}$ (Figura 7). Sin embargo, es notable que tan sólo las 10 partículas de mayores largos, superiores a $73 \mu \mathrm{m}$, representan el $27 \%$ del largo total acumulado de las partículas. Según las gráficas de largo por ancho se aprecia la presencia de partículas de oro grueso que aportan más del $40 \%$ de oro y que producen un efecto pepita importante (presencia de oro grueso). Las relaciones largo por ancho son aproximadamente de $1.7 / 1$ a $2 / 1$.

En la muestra del barreno de la Zona B se identificaron 40 partículas de oro (Figura 7). La media de los largos de las partículas es de $30 \mu \mathrm{m}$ y la media de los anchos es de $20 \mu \mathrm{m}$. Todas las partículas de oro son menores a $60 \mu \mathrm{m}$. La relación de largo por ancho predominante en las partículas es de 1.7/1. 
A Composición mineralógica $(\%$ peso $)$
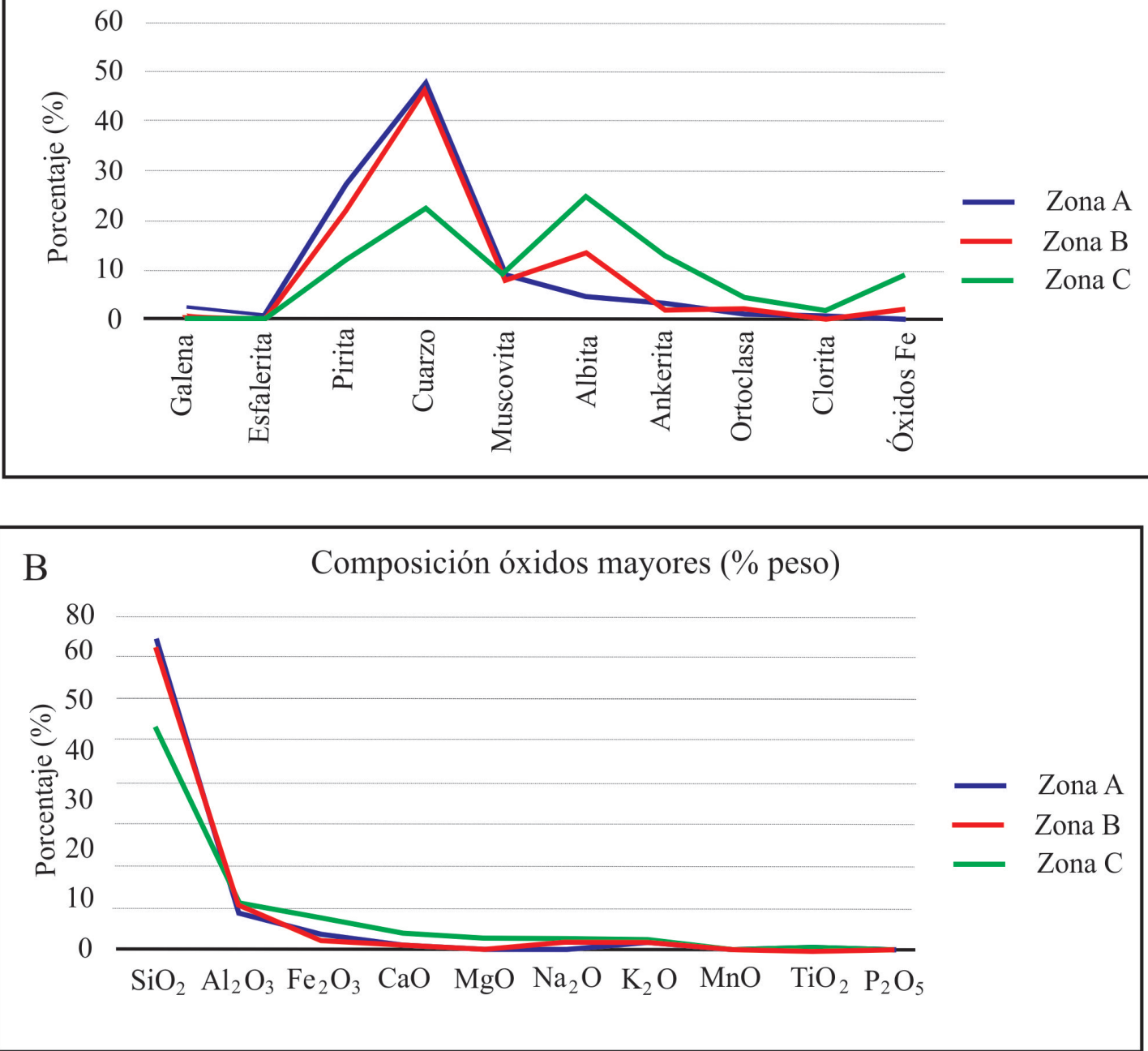

C Composición óxidos mayores (\% peso)

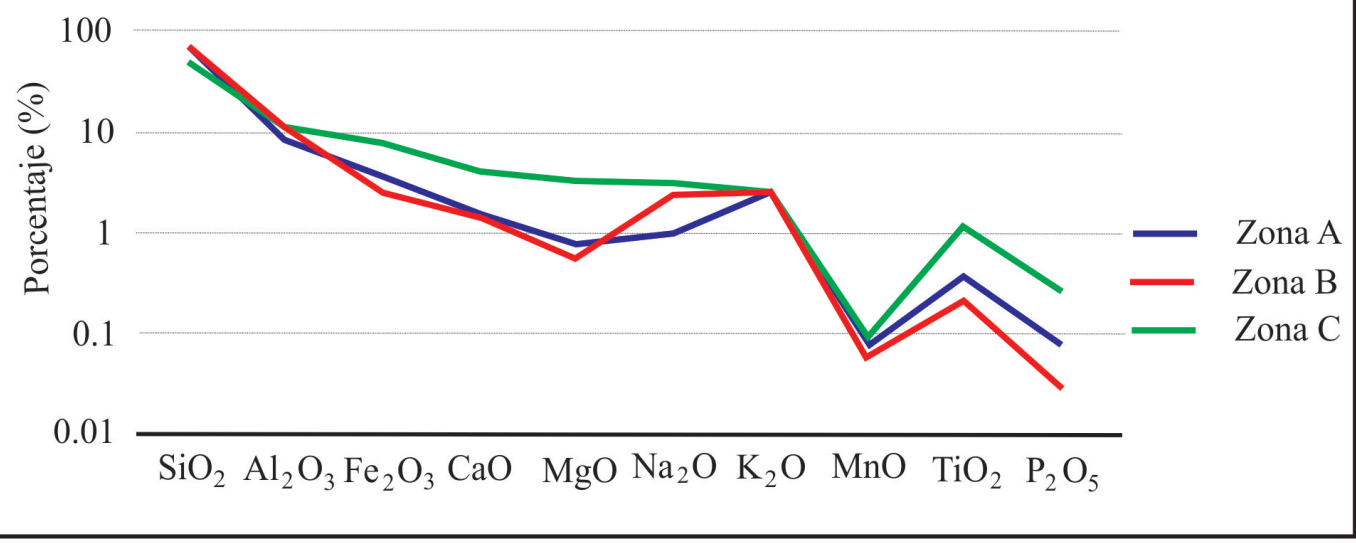

Figura 6 A. Composición modal de los concentrados gravimétricos obtenidos con Mineral Liberation Analizer (MLA); B. Composición en óxidos mayores de los compósitos antes de la concentración gravimétrica; C. Misma que la anterior en escala logarítmica. 
Tabla 4. Composición en óxidos mayores de los tramos y compósitos de las muestras antes de la concentración gravimétrica.

\begin{tabular}{|c|c|c|c|c|c|c|c|c|c|c|}
\hline Muestras & \multicolumn{10}{|c|}{ Elementos Mayores } \\
\hline Zona $A$ & $\mathrm{SiO}_{2}$ & $\mathrm{Al}_{2} \mathrm{O} 3$ & $\mathrm{Fe}_{2} \mathrm{O}_{3}$ & $\mathrm{CaO}$ & $\mathrm{MgO}$ & $\mathrm{Na}_{2} \mathrm{O}$ & $\mathrm{K}_{2} \mathrm{O}$ & $\mathrm{MnO}$ & $\mathrm{TiO}_{2}$ & $\mathrm{P}_{2} \mathrm{O}_{5}$ \\
\hline HPG-121 M-1A & 75.7 & 8.51 & 3.64 & 2.11 & 0.7 & 0.63 & 2.35 & 0.06 & 0.29 & 0.06 \\
\hline$H P G-187 M-1 A$ & 75.2 & 10.03 & 3.08 & 1.3 & 0.61 & 1.3 & 2.83 & 0.06 & 0.25 & 0.04 \\
\hline HPG-190 M-1A & 71.87 & 11.44 & 3.39 & 1.77 & 0.69 & 1.77 & 2.84 & 0.09 & 0.35 & 0.07 \\
\hline$B D S-13-C M-1 A$ & 79.46 & 9.05 & 2.73 & 0.41 & 0.31 & 0.93 & 2.59 & 0.03 & 0.12 & 0.02 \\
\hline BDS-13-H M-1A & 77.53 & 9.73 & 4.43 & 0.39 & 0.39 & 0.07 & 3.23 & 0.03 & 0.41 & 0.13 \\
\hline$B D S-25-D M-1 A$ & 65.98 & 6.25 & 9.17 & 3.12 & 1.83 & 0.46 & 1.49 & 0.11 & 1.14 & 0.27 \\
\hline ZONA A & 74.64 & 9.1 & 3.91 & 1.61 & 0.77 & 0.99 & 2.46 & 0.07 & 0.39 & 0.08 \\
\hline Promedio & 74.34 & 9.16 & 4.34 & 1.53 & 0.76 & 0.88 & 2.54 & 0.06 & 0.42 & 0.1 \\
\hline Muestras & \multicolumn{10}{|c|}{ Elementos Mayores } \\
\hline Zona $B$ & $\mathrm{SiO}_{2}$ & $\mathrm{Al}_{2} \mathrm{O} 3$ & $\mathrm{Fe}_{2} \mathrm{O}_{3}$ & $\mathrm{CaO}$ & $\mathrm{MgO}$ & $\mathrm{Na}_{2} \mathrm{O}$ & $\mathrm{K}_{2} \mathrm{O}$ & $\mathrm{MnO}$ & $\mathrm{TiO}_{2}$ & $\mathrm{P}_{2} \mathrm{O}_{5}$ \\
\hline HPG-28 M-1A & 72.86 & 11.79 & 2.57 & 1.67 & 0.6 & 2.79 & 2.56 & 0.06 & 0.29 & 0.03 \\
\hline$H P G-54-M 1 A$ & 73.92 & 13.02 & 3.08 & 0.33 & 0.44 & 1.53 & 3.45 & 0.04 & 0.3 & 0.04 \\
\hline ZONA B & 73.06 & 12.05 & 2.6 & 1.49 & 0.58 & 2.56 & 2.8 & 0.06 & 0.22 & 0.03 \\
\hline Promedio & 73.28 & 12.29 & 2.75 & 1.16 & 0.54 & 2.29 & 2.94 & 0.05 & 0.27 & 0.03 \\
\hline Muestras & \multicolumn{10}{|c|}{ Elementos Mayores } \\
\hline Zona C & $\mathrm{SiO}_{2}$ & $\mathrm{Al}_{2} \mathrm{O} 3$ & $\mathrm{Fe}_{2} \mathrm{O}_{3}$ & $\mathrm{CaO}$ & $\mathrm{MgO}$ & $\mathrm{Na}_{2} \mathrm{O}$ & $\mathrm{K}_{2} \mathrm{O}$ & $\mathrm{MnO}$ & $\mathrm{TiO}_{2}$ & $\mathrm{P}_{2} \mathrm{O}_{5}$ \\
\hline$H P G-08 M-1 A$ & 54.76 & 11.63 & 8.31 & 4.58 & 3.6 & 2.81 & 2.24 & 0.1 & 1 & 0.11 \\
\hline HPG-40 M-1A & 43.86 & 11.85 & 11 & 5.9 & 4.79 & 4.25 & 1.69 & 0.13 & 1.72 & 0.49 \\
\hline$H P G-47-M 1 A$ & 63.87 & 11.62 & 5.34 & 2.7 & 1.96 & 2.41 & 4.01 & 0.06 & 0.66 & 0.13 \\
\hline ZONA C & 53.48 & 11.78 & 8 & 4.34 & 3.41 & 3.24 & 2.68 & 0.1 & 1.21 & 0.27 \\
\hline Promedio & 53.99 & 11.72 & 8.16 & 4.38 & 3.44 & 3.18 & 2.66 & 0.1 & 1.15 & 0.25 \\
\hline
\end{tabular}

\subsubsection{CARACTERÍSTICAS DEL ORO IDENTIFICADO} CON MLA

Se analizaron tres secciones pulidas para cada muestra de concentrado (9 briquetas en total).

En total, fueron identificadas 109 partículas de oro en las 9 briquetas analizadas, de las cuales 64 partículas correspondieron a oro con una composición de electrum y 14 a petzita $\left(\mathrm{Ag}_{3} \mathrm{AuTe}_{2}\right.$ con $25 \%$ de $\mathrm{Au}$ ). Por otra parte, también fue identificada stutzita (AgTe). El contenido de plata detectado en las partículas de oro electrum se encuentra en cantidades variables de entre $20 \%$ y $30 \%$ aproximadamente (el electrum contiene $>20 \%$ de $\mathrm{Ag}$ ). Las principales asociaciones texturales de las partículas de oro identificadas consisten en inclusiones en pirita o en diversas gangas como cuarzo, albita, ortoclasa o ankerita; también se observaron algunas asociaciones como películas en el borde de la pirita. La composición promedio del oro es de $74 \%$ de $\mathrm{Au}$ y $26 \%$ de $\mathrm{Ag}$, con una fórmula empírica calculada de $\mathrm{Au}_{0.6} \mathrm{Ag}_{0.4}$.

En la Tabla 5 y figuras 10, 11 y 12 se muestran las dimensiones y el tipo de asociación que presentan las partículas de oro identificadas para cada muestra. Se puede observar que en las zonas A y B el oro se encuentra en forma de electrum pero en la zona $\mathrm{C}$ el oro puede provenir, tanto de electrum, como de petzita.

Del total de las partículas de oro identificadas para las 3 zonas, la mitad de las partículas tienen largos menores a $30 \mu \mathrm{m}$ y anchos menores a 9.3 $\mu \mathrm{m}$ (Figura 10). Sin embargo, tan sólo 5 partículas, de largos superiores a $73 \mu \mathrm{m}$, son capaces de representar el 35\% del largo total acumulado de las partículas; de la misma manera, 5 partículas, de ancho superior a $18 \mu \mathrm{m}$, son capaces de representar el 25\% del ancho total acumulado de 

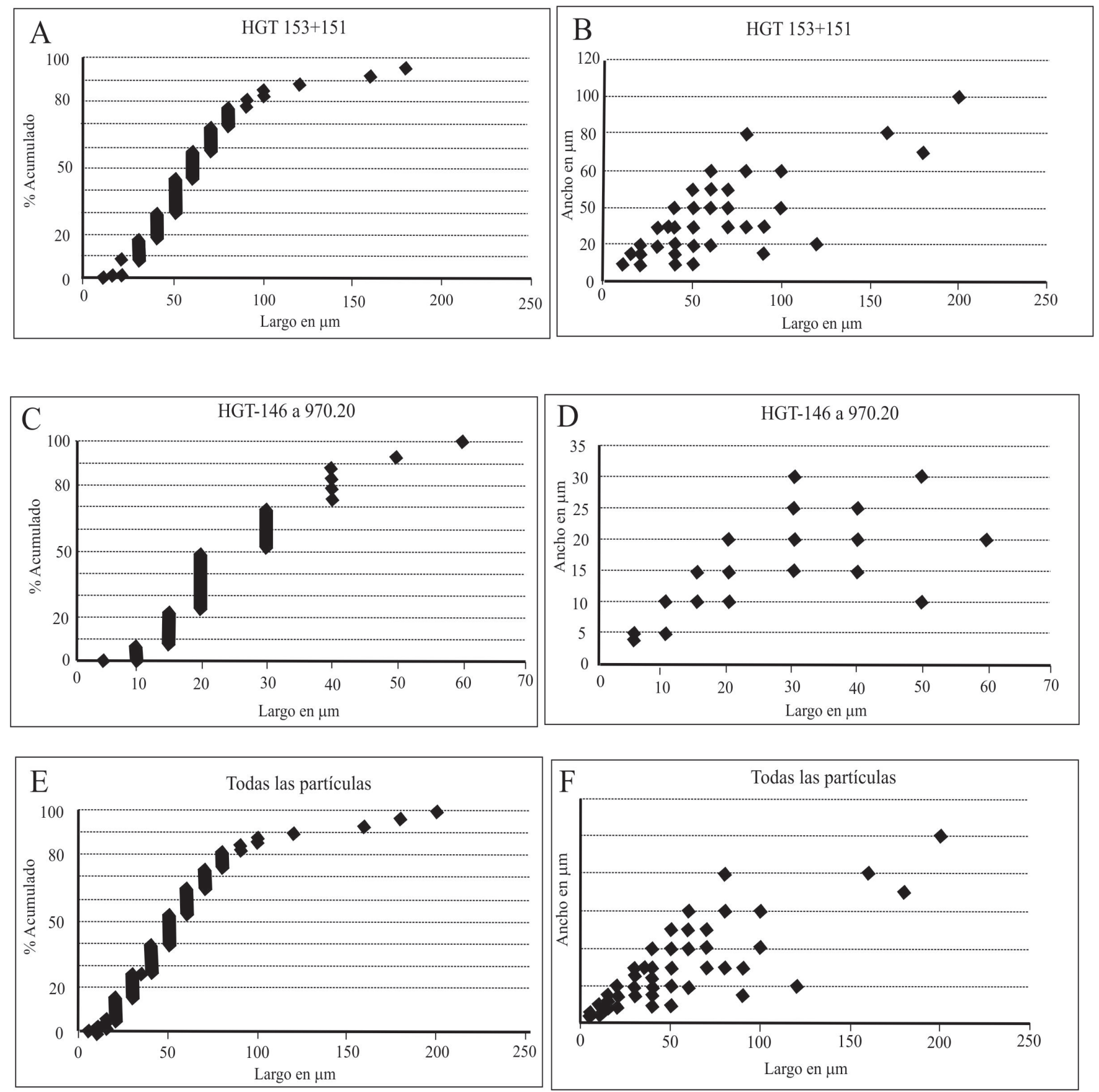

Figura 7 A. Porcentaje acumulado para los largos de las partículas de oro de los barrenos HGT-153@1170.80 m y HGT151@1316.25 m. B. Largo por ancho de las partículas de oro de los barrenos HGT-153@1170.80 m y HGT-151@1316.25 m (observar la importante cantidad de partículas de oro grueso). C. Largo de las partículas de oro observadas en el Barreno HGT-146@970.20 m D. Largo por ancho de las partículas de oro para HGT-146@970.20 m. E. Largo para todas las partículas de oro observadas en los barrenos anteriores. F. Largo por ancho de las partículas de oro para todas las partículas de oro de las muestras de los barrenos. 


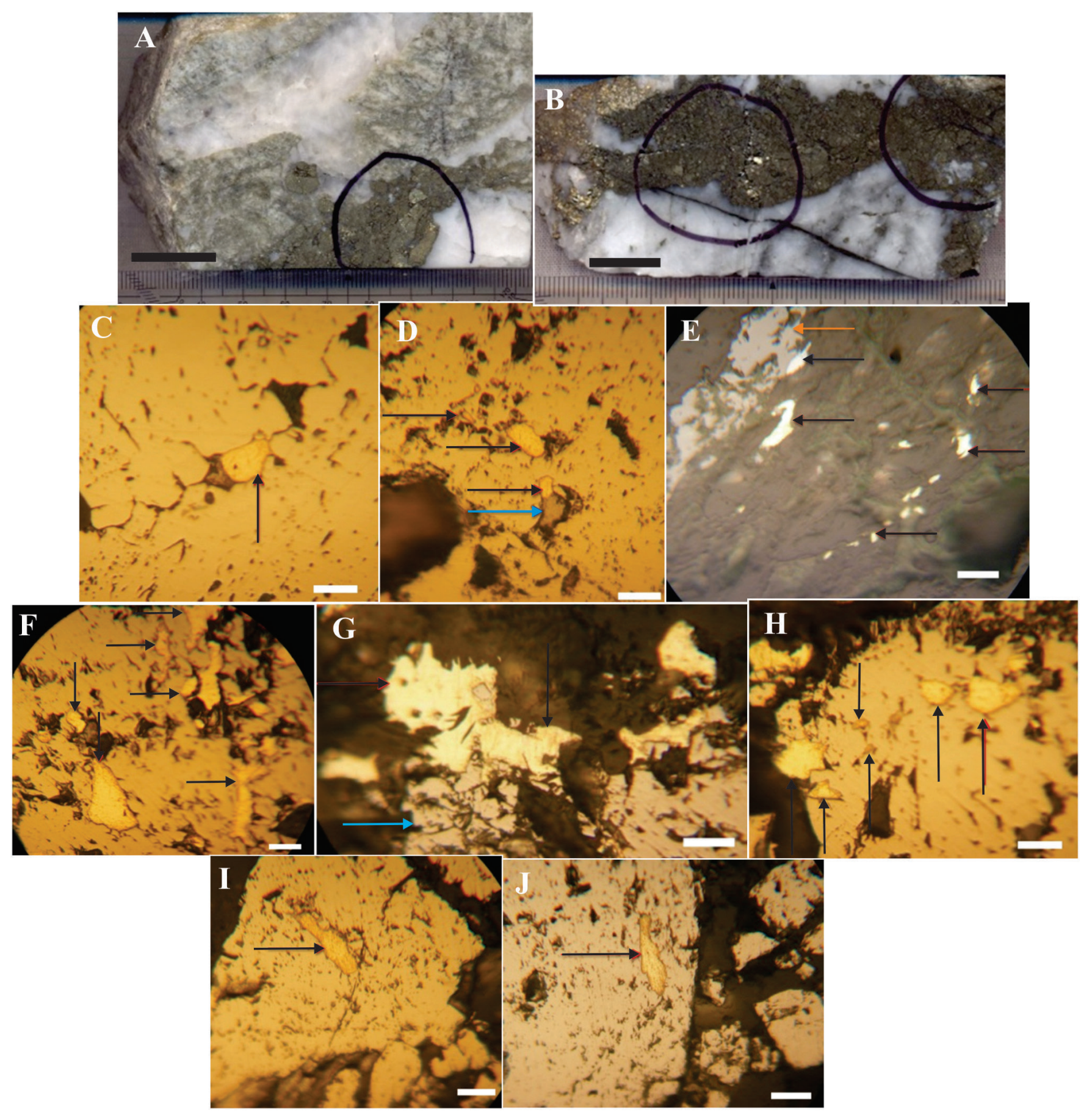

Figura 8 A. Muestra HGT-146 a 970.20 m (se indica la zona de la sección pulida). B. Muestra HGT-151 a 1316.25 m (se indican zonas de las secciones pulidas). C. Placa de oro entre pirita (LR). D. Se observan inclusiones de oro en pirita (LR). E. Observar al menos 10 partículas de oro en ganga no metálica (LR). F. Abundante oro en pirita (LR). G. Placas de oro con pirita (LR). H. Placas de oro en pirita (LR). I. Inclusión de oro en pirita (LR). J. Inclusión de oro en pirita (LR). Explicación: LR: Luz Reflejada; flecha negra: oro, flecha azul: galena, flecha naranja: esfalerita; la escala representa para $A$ y B: $2 \mathrm{~cm}$, para C, D, E, F, G, H, I y J: $50 \mu \mathrm{m}$. 


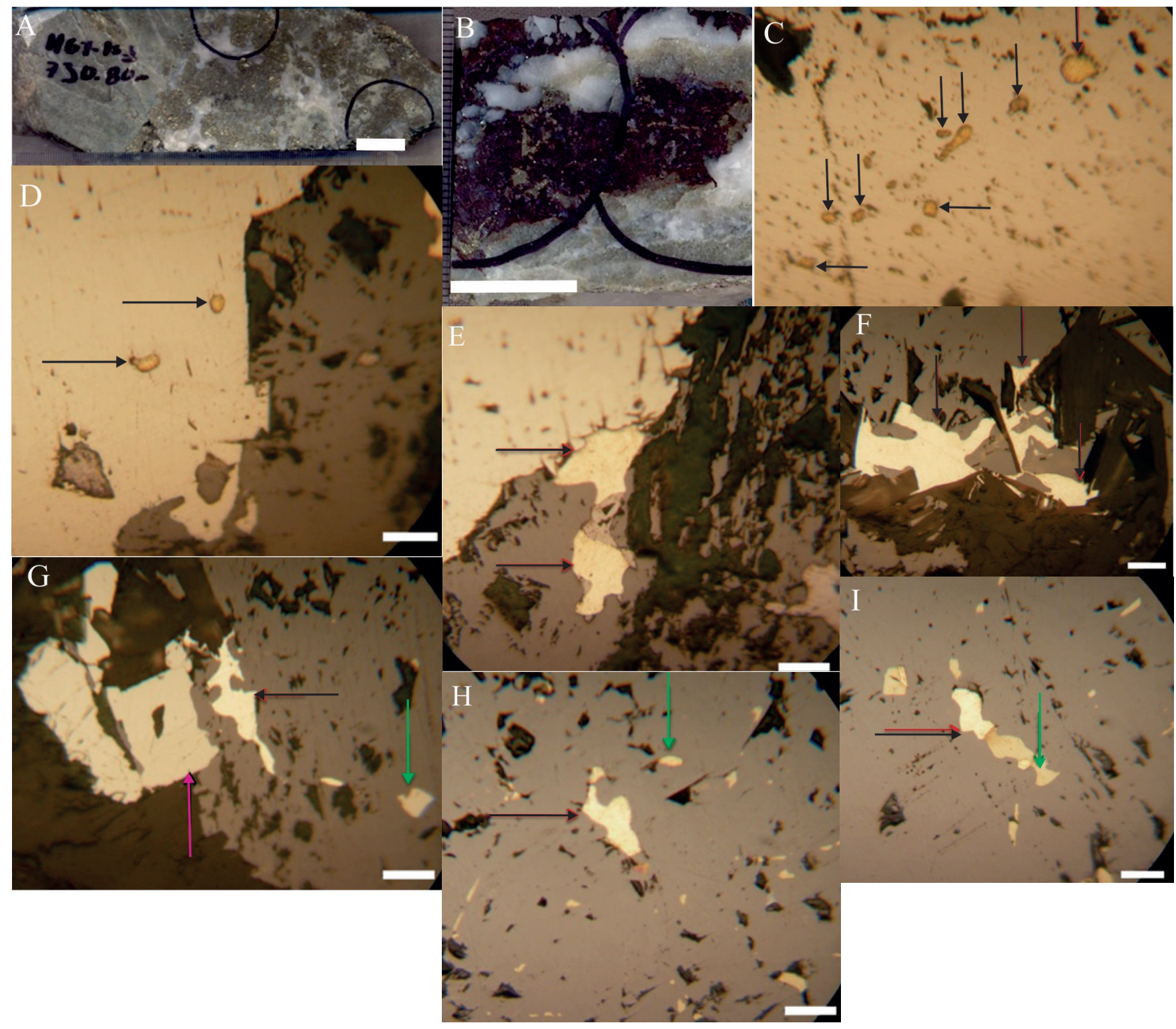

Figura 9 A. Muestra HGT-153 a 730.80 m (se indican zonas de las secciones pulidas). B. Muestra HGT-153 a $1170.80 \mathrm{~m}$ (se indican zonas de las secciones pulidas), la parte café corresponde a esfalerita. C. Se observan varias partículas de oro incluidas en pirita, (LR). D. Inclusiones de oro en pirita, (LR). E. Partículas de oro asociado a esfalerita y galena (LR). F. Oro asociado a esfalerita y sericita (LR). G, H e I. Oro incluido en esfalerita e inclusiones de calcopirita (LR). Explicación: LR: luz reflejada; flecha negra: oro, flecha verde: calcopirita, flecha roja: pirita. Escalas para A y B: $2 \mathrm{~cm}$, para C, D, E, F, G, H e I: $50 \mu \mathrm{m}$.

partículas. La tendencia del mayor aporte de oro a partir de partículas gruesas (efecto pepita) es evidente en las zonas A y B, como puede observarse en las gráficas. Sin embargo, todas las partículas de oro identificadas en la Zona $\mathrm{G}$ son inferiores a $22 \mu \mathrm{m}$, por lo que se supone que el efecto pepita en esta zona está más amortiguado, aunque la presencia en la misma de pequeñas proporciones de oro en petzita debería representar problemas de análisis de Au soluble en CNNa y, por ende, de recuperación por cianuración.

Según las gráficas de largo por ancho se aprecian 2 poblaciones de oro: unas de oro grueso que aportan la mayor cantidad de oro y que producen un efecto pepita importante, con relaciones largo por ancho de 2.5/1 a 10/1 y otra población con 
mayor número de granos homogéneos, en general, presente como inclusiones y de una relación largo por ancho más o menos constante de 2/1. El efecto pepita se advierte claramente en las zonas A y B mas no así en la Zona $\mathrm{C}$ en la que el oro identificado es más fino.

La Tabla 5 y las figuras 11 y 12 muestran claramente la manera de presentación del oro, así como las relaciones texturales con los otros minerales.

\section{Cálculo de recuperaciones de oro para un modelo geometalúrgico}

El modelo geometalúrgico debería permitir definir los mejores destinos para el mineral de alta ley de la zona de sulfuros primarios de la mina La Herradura. En el Anexo 3 se enlistan algunas premisas básicas tomadas en cuenta para la realización del modelo.

Las constantes de cálculo de recuperaciones metalúrgicas para cada una de las zonas se hicieron tomando en cuenta el tamaño y las texturas de todas las partículas con oro reconocidas con Mineral Liberation Analizer (MLA). Se consideró como oro recuperable en cianuración dinámica a toda partícula de oro libre o dispuesta en los bordes de sulfuros o en microfracturas en sulfuros (es decir, percolable por cianuración). Por otra parte, se consideró como oro no recuperable a todas aquellas partículas de oro observadas como inclusiones en pirita y de un tamaño inferior al tamaño de molienda actual de cianuración dinámica (-0.075 $\mathrm{mm}$, que fue el diseño de planta). También se consideró como oro no recuperable aquel que se encuentra en forma de petzita $\left(\mathrm{Ag}_{3} \mathrm{AuTe}_{2}\right)$, según la experiencia en minas de este tipo y en datos de la literatura (Marsden y House, en Goodall y Scales, 2007). Estos cálculos nos dan como resultado una "metalurgia física", en la cual no se consideran pruebas metalúrgicas experimentales de cianuración.
De una manera general, las constantes de recuperación calculadas provienen de las sumas largo por ancho del oro no recuperable, así como del oro presente en telururos dividido entre la suma de largo por ancho del oro total. Los cálculos comprenden todas las partículas de las 3 secciones pulidas por cada zona: A, B y C. Las constantes de recuperación calculadas a partir de MLA fueron, para Zona A: 92\%, para Zona B: 79\% y para Zona C: 84\%. En el caso de la Zona C se calculó que el $11 \%$ del oro se encuentra en petzita.

Las otras constantes de recuperación experimentales a partir de pruebas de cianuración en botellas fueron, a un tamaño de partícula de $-0.84 \mathrm{~mm}$, para la Zona A: 87\%, con una ley de cabeza ensayada de $15.5 \mathrm{~g} / \mathrm{t}$ de Au y una ley de cabeza calculada de $16.7 \mathrm{~g} / \mathrm{t}$ de Au; para la Zona B: 92\%, con una ley de cabeza ensayada de $5.7 \mathrm{~g} / \mathrm{t}$ de Au y una ley de cabeza calculada de $4.4 \mathrm{~g} / \mathrm{t}$ de Au, y para la Zona C: 90\%, con una ley de cabeza ensayada de $1.5 \mathrm{~g} / \mathrm{t}$ de Au y una ley de cabeza calculada de 3.4 $\mathrm{g} / \mathrm{t}$ de $\mathrm{Au}$. Finalmente, las constantes de recuperación experimentales a un tamaño de partícula de -0.075 mm fueron, para la Zona A: 94\%, con una ley de cabeza ensayada de $15.5 \mathrm{~g} / \mathrm{t}$ de Au y una ley de cabeza calculada de $15.3 \mathrm{~g} / \mathrm{t}$ de $\mathrm{Au}$; para la Zona B: 98\%, con una ley de cabeza ensayada de $5.7 \mathrm{~g} / \mathrm{t}$ de Au y una ley de cabeza calculada de $6.0 \mathrm{~g} / \mathrm{t}$ de Au, y para la Zona C: 91\%, con una ley de cabeza ensayada de $1.5 \mathrm{~g} / \mathrm{t}$ de Au y una ley de cabeza calculada de $2.4 \mathrm{~g} / \mathrm{t}$ de $\mathrm{Au}$.

Se realizó la cubicación de cada una de las zonas utilizando el modelo de bloques actual. Las leyes medias por bloque se modificaron a partir de las constantes calculadas por MLA u obtenidas por experimentación. Cada bloque del modelo mide 15x15x8 m y el modelo para efectuar el cálculo se basa en el software TSS Studio propiedad de Newmont, que es el que utiliza la empresa concesionaria del depósito para sus cálculos de recursos. Los contenidos totales de oro y los contenidos de oro recuperado se muestran en el Anexo 4, así 
Tabla 5. Características de las partículas de oro observadas con MLA.

\begin{tabular}{|c|c|c|c|}
\hline c & $\operatorname{Largo}(\mu \mathrm{m})$ & Ancho( $\mu m)$ & Asociación \\
\hline 1 ZAPK1 & 162.3 & 18.3 & Oro electrum en borde de pirita \\
\hline 2 ZAPK1 & $17.7,10.4$ & $6.5,8.3$ & Inclusiones de oro electrum en pirita + cuarzo \\
\hline 3 ZAPK1 & 7 & 4.8 & Inclusión de oro electrum en pirita + cuarzo \\
\hline 4 ZAPK1 & 224.3 & 77.9 & Partícula binaria de oro electrum y pirita \\
\hline 5 ZAPK1 & $17.2,3.7$ & $7.0,1.9$ & Inclusiones de oro electrum en pirita + cuarzo \\
\hline 6 ZAPK1 & 16 & 9.7 & Inclusión de oro electrum en pirita + ankerita \\
\hline 7 ZAPK1 & $7.6,1.2$ & $4.1,0.9$ & Inclusiones de oro electrum en pirita + cuarzo \\
\hline 8 ZAPK1 & 4.4 & 3.2 & Inclusión de oro electrum en pirita + galena \\
\hline 1 ZAPK2 & $19.8,11.9$ & $15.1,12.2$ & Inclusiones de oro electrum en pirita \\
\hline 2 ZAPK2 & 55.8 & 8 & Partícula de oro electrum binaria con pirita \\
\hline 3 ZAPK2 & 16 & 14 & Partícula de oro electrum binaria con pirita \\
\hline 4 ZAPK2 & $14.8,7.1$ & $4.4,3.0$ & Partículas libres de oro electrum \\
\hline 5 ZAPK2 & 10.5 & 9.3 & Partícula ternaria de pirita, galena y oro electrum \\
\hline 6 ZAPK2 & $10.2,5.3$ & $8.1,3.1$ & $\begin{array}{l}\text { Inclusiones de oro electrum en partícula de pirita y } \\
\text { esfalerita }\end{array}$ \\
\hline 7 ZAPK2 & 8 & 4.5 & Inclusión de oro electrum en pirita + cuarzo \\
\hline 8 ZAPK2 & $13.1,11.0$ & $7.7,3.5$ & Inclusiones de oro electrum en pirita \\
\hline 9 ZAPK2 & 12.8 & 3.2 & Inclusión de oro electrum en pirita \\
\hline 10 ZAPK2 & 33.2 & 16.9 & $\begin{array}{l}\text { Oro electrum en partícula multifase de pirita, galena y } \\
\text { esfalerita }\end{array}$ \\
\hline 11 ZAPK2 & 33.4 & 9.3 & Partícula libre de oro electrum \\
\hline 12 ZAPK2 & 5.7 & 2.8 & Oro electrum en borde de pirita + galena \\
\hline 13 ZAPK2 & $12.5,8.0$ & $8.7,6.4$ & Inclusión de oro electrum en pirita + galena \\
\hline 14 ZAPK2 & 16.8 & 9.1 & Inclusión de oro electrum en esfalerita +pirita \\
\hline 15 ZAPK2 & $10.8,7.4,1.9$ & $5.2,5.9,1.9$ & Inclusiones de oro electrum en pirita + muscovita \\
\hline 16 ZAPK2 & 16.6 & 5.6 & Inclusión de oro electrum en pirita + galena \\
\hline 17 ZAPK2 & 7.1 & 5.2 & Oro electrum en borde de pirita + galena \\
\hline 18 ZAPK2 & 5.5 & 4.4 & Inclusión de oro electrum en pirita + galena \\
\hline 19 ZAPK2 & 3.5 & 1.6 & $\begin{array}{l}\text { Oro electrum en partícula multifase de pirita, } \\
\text { muscovita y galena }\end{array}$ \\
\hline 1 ZAPK3 & 200 & 80 & Partícula ternaria de oro electrum, pirita y galena \\
\hline 2 ZAPK3 & $\begin{array}{l}17.6,15.7 \\
11.6\end{array}$ & $3.5,7.1,5.7$ & $\begin{array}{l}\text { Inclusiones de oro electrum en pirita, muscovita y } \\
\text { galena }\end{array}$ \\
\hline 3 ZAPK3 & $\begin{array}{l}19.5,12.5 \\
6.9,5.8\end{array}$ & $3.0,1.9,2.5,3.6$ & $\begin{array}{l}\text { Múltiples inclusiones de oro electrum en pirita }+ \\
\text { ortoclasa }\end{array}$ \\
\hline 4 ZAPK3 & 5.6 & 5.1 & Inclusión de oro electrum en pirita + galena \\
\hline 5 ZAPK3 & $30.2,16.2$ & $11.3,10.4$ & $\begin{array}{l}\text { Inclusiones de oro electrum en pirita, esfalerita y } \\
\text { galena }\end{array}$ \\
\hline 6 ZAPK3 & 32.2 & 17.1 & Inclusión de oro electrum en pirita \\
\hline 7 ZAPK3 & 13.6 & 5.8 & Partícula binaria de pirita y oro electrum \\
\hline 8 ZAPK3 & 6 & 3.2 & Inclusión de oro electrum en pirita \\
\hline
\end{tabular}



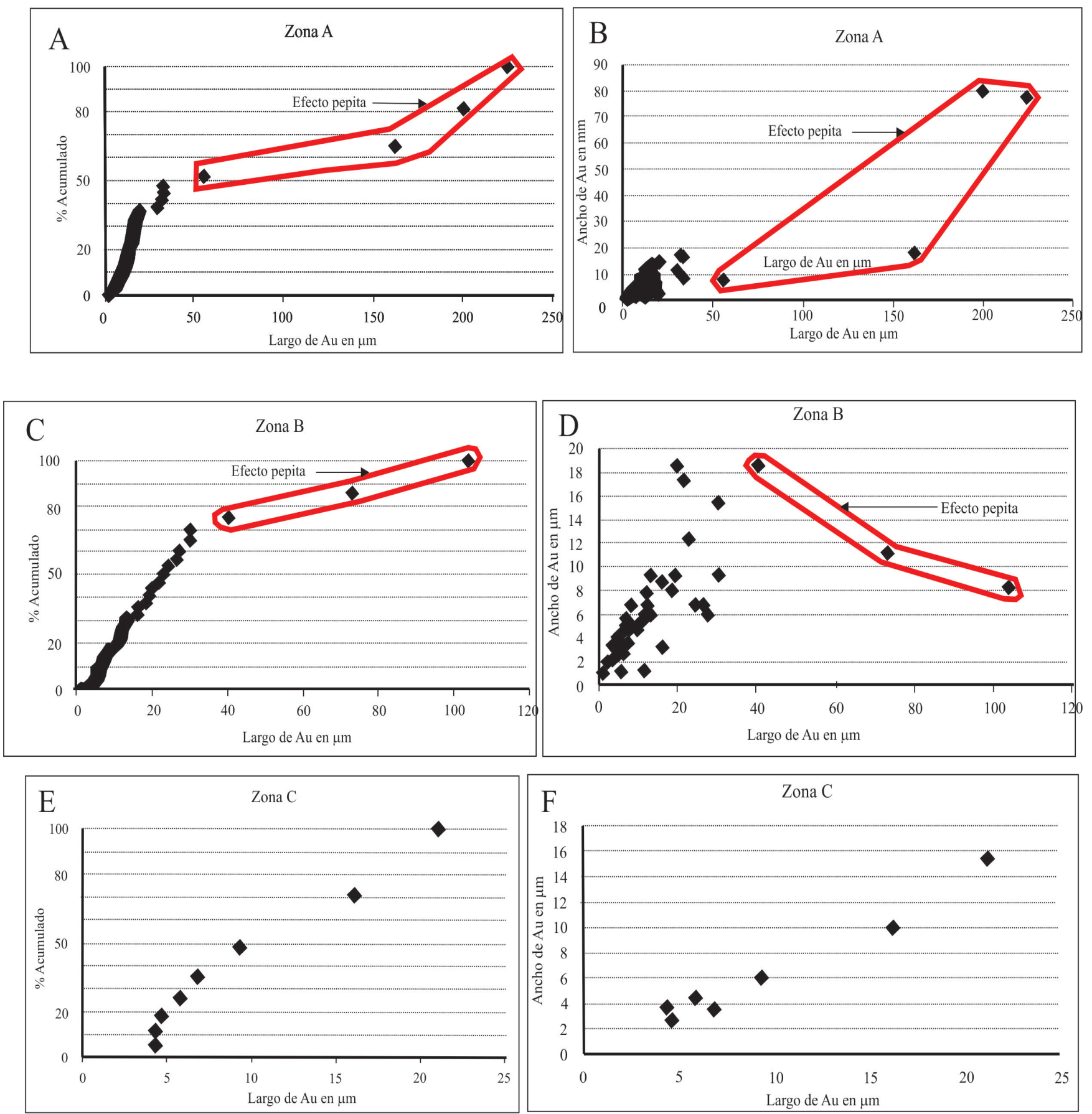

Figura 10 A. Porcentaje acumulado de los largos de las partículas de oro de la Zona A (observar que cuatro partículas representan el $48 \%$ del largo total acumulado de las partículas). B. Largo por ancho de las partículas de oro para la Zona A (observar las cuatro partículas que provocan el "efecto pepita"). C. Porcentaje acumulado de los largos de las partículas de oro de la Zona B (observar que tres partículas representan el $25 \%$ del largo total acumulado de las partículas). D. Largo por ancho de las partículas para la Zona B (observar las partículas que provocan el "efecto pepita"). E. Porcentaje acumulado de los largos de las partículas de oro de la Zona C (no se aprecia "efecto pepita"). F. Largo por ancho de las partículas para la Zona C. 

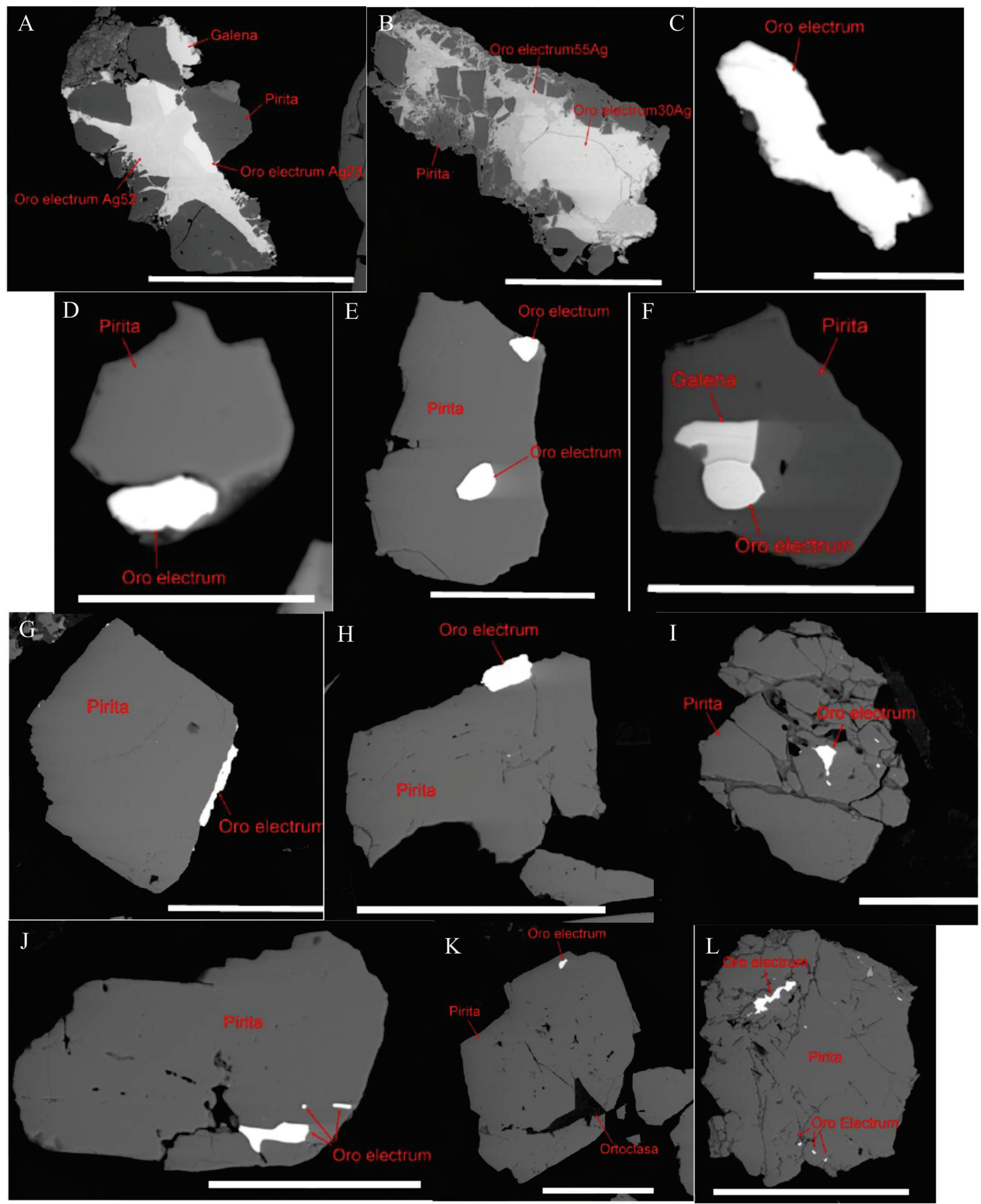

Figura 11 A. Zona A; partícula mixta de oro (electrum) de $52 \%$ de Ag con otra de $23 \%$ de Ag introducida en pirita. B. Zona A; oro (electrum) de $55 \%$ Ag y otra de $30 \%$ Ag asociado con pirita. C. Zona A; partícula de oro (electrum) libre. D. Zona A; oro (electrum) asociado con pirita. E. Zona A; inclusiones de oro (electrum) en pirita. F. Zona A; inclusiones de oro (electrum) y galena en pirita. G. Zona A; oro (electrum) en película bordeando pirita. H. Zona B; oro (electrum) en el borde de pirita. I. Zona B; inclusiones de oro en pirita. J. Zona B; partículas de oro (electrum) en pirita. K. Zona B; inclusión de oro (electrum) en pirita. L. Zona B; inclusiones de oro (electrum) en pirita. Escalas: $200 \mu \mathrm{m}$ para A y H; $100 \mu \mathrm{m}$ para B, E, I y J; $30 \mu \mathrm{m}$ para C y D; $50 \mu \mathrm{m}$ para F; $400 \mu \mathrm{m}$ para G; $500 \mu \mathrm{m}$ para K; $300 \mu \mathrm{m}$ para L. 


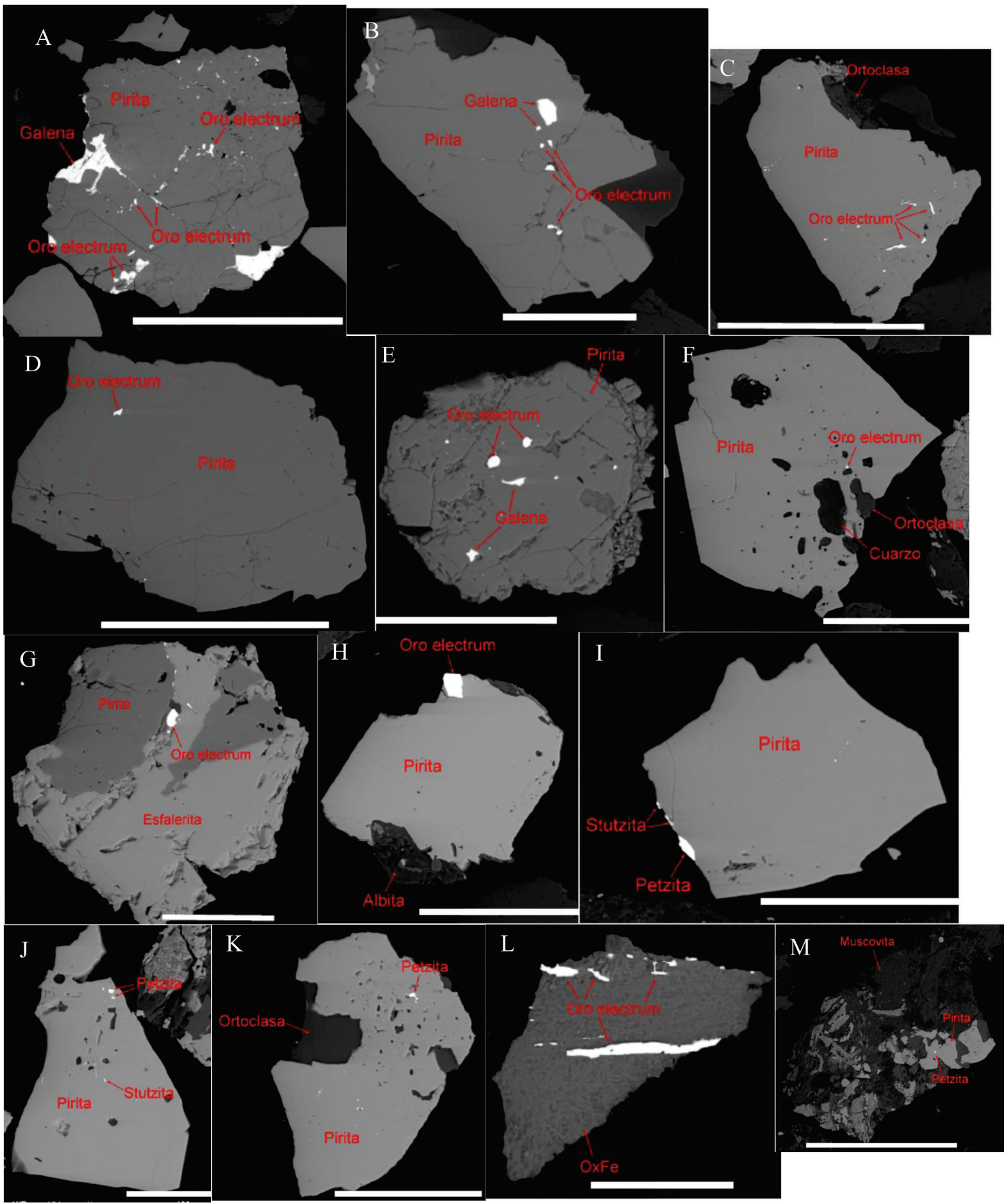

Figura 12 A. Zona B; inclusiones de oro (electrum) y otras numerosas inclusiones de galena en pirita. B. Zona B; inclusiones de oro (electrum) y galena en pirita. C. Zona A; partículas de oro (electrum) incluidas en pirita. D. Zona B; inclusión de oro (electrum) en pirita. E. Zona B; partículas de oro (electrum) y galena incluidas en pirita. F. Zona C; inclusión de oro (electrum) en partícula de pirita-cuarzoortoclasa. G. Zona A; partículas de oro (electrum) entre esfalerita y pirita. H. Zona C; partícula de oro (electrum) en el borde de piritaalbita. I. Zona C; petzita y stutzita en los bordes pirita. J. Zona C; inclusiones de petzita y stutzita en pirita. K. Zona C; inclusión de petzita en partícula de pirita-ortoclasa. L. Zona B; oro (electrum) introducido en partícula de óxidos de fierro (probable pirita original). M. Zona C; inclusión de petzita en pirita con muscovita y otros. Escalas: $500 \mu \mathrm{m}$ para A; $100 \mu \mathrm{m}$ para B, E, G, H, I, J, K, L; $200 \mu \mathrm{m}$ para C, D, F; 400 $\mu \mathrm{m}$ para $\mathrm{M}$. 
como en la Tabla 6 y Figura 13, resumiéndose de la siguiente manera: cálculos por zona y de acuerdo con diferentes leyes mínimas de corte (cutoff; ; cálculos utilizando las constantes de recuperación obtenidas por MLA y cálculos utilizando las constantes de recuperación de las pruebas experimentales a -0.84 mm y utilizando las constantes de recuperación experimentales a $-0.075 \mathrm{~mm}$.

\section{Discusión y conclusiones}

La utilización de Mineral Liberation Analizer (MLA) permitió reconstruir la mineralogía de los concentrados gravimétricos, la cual se compone principalmente de cuarzo, pirita, feldespato y muscovita (sericita). Las composiciones de las zonas A y B son muy similares, pero el concentrado de la Zona
C es mucho más pobre en cuarzo y pirita, y se enriquece en albita, en magnetita y en ankerita.

Existe congruencia entre la mineralogía de los concentrados y la composición en óxidos mayores de los compósitos que se relaciona con la mineralogía de los mismos. Es evidente que el déficit de $\mathrm{SiO}_{2}$ para la Zona $\mathrm{C}$ en el compósito se relaciona con la menor cantidad de cuarzo libre, ya sea en vetillas o en la roca encajonante. El incremento en $\mathrm{Na}_{2} \mathrm{O}$ en el compósito se debe a la presencia de albita; la abundancia en $\mathrm{Fe}_{2} \mathrm{O}_{3}$ es debido a la cantidad de magnetita-hematita presentes, y los valores de $\mathrm{CaO}$ y $\mathrm{MgO}$ se pueden correlacionar con los contenidos de ankerita $(\mathrm{CaO})$ y quizás alguna parte con la presencia de clorita $(\mathrm{MgO})$.

En relación con el contenido de oro en las muestras, existen serias diferencias entre las leyes de cabeza ensayadas para las pruebas gravimétricas

Tabla 6. Onzas totales de oro, onzas recuperables y diferencias porcentuales entre oro recuperable calculado y oro recuperable experimental.

\begin{tabular}{|l|c|c|c|c|c|c|c|c|}
\hline & $\begin{array}{c}(1) \\
\text { Oz totales }\end{array}$ & $\begin{array}{c}(2) \\
\text { Rec MLA }\end{array}$ & $\begin{array}{c}(3) \\
\text { RecMLAyEx } \\
-0.84 \mathrm{~mm}\end{array}$ & $\begin{array}{c}(4) \\
\text { RecMLAyEx } \\
-0.075 \mathrm{~mm}\end{array}$ & $\begin{array}{c}(5) \\
\operatorname{RecExp} \\
-0.84 \mathrm{~mm}\end{array}$ & $\begin{array}{c}(6) \\
\text { RecExp } \\
-0.075 \mathrm{~mm}\end{array}$ & $(7)$ & $(8)$ \\
\hline Zona A & $4,470,276$ & $4,112,654$ & $4,058,563.58$ & $4,128,389.29$ & $3,889,140$ & $4,202,060$ & 0.04 & -0.017 \\
\hline Zona B & $5,833,173$ & $4,608,206$ & $4,734,203.21$ & $4,788,335.05$ & $5,366,519$ & $5,716,509$ & -0.13 & -0.193 \\
\hline Zona C & $3,988,875$ & $3,350,655$ & $3,376,981.58$ & $3,382,685.67$ & $3,589,988$ & $3,629,876$ & -0.06 & -0.073 \\
\hline Totales & $14,292,324$ & $12,071,515$ & $12,169,748.36$ & $12,299,410.01$ & $12,845,647$ & $13,548,445$ & -0.05 & -0.101 \\
\hline
\end{tabular}

1 Onzas totales de oro calculadas.

2 Onzas recuperables de oro calculadas con constantes de MLA.

$3{ }^{A}$ Onzas recuperables calculadas con constantes de MLA para Au recuperado con gravimetría y e experimentalmente por cianuración a un tamaño de $-0.84 \mathrm{~mm}$.

4 BOnzas recuperables con constantes de MLA para Au recuperado con gravimetría y el resto para experimentalmente por cianuración a un tamaño de $-0.075 \mathrm{~mm}$.

5 Onzas recuperables por cianuración dinámica a un tamaño de partícula de $-0.84 \mathrm{~mm}$.

6 Onzas recuperables por cianuración dinámica a un tamaño de partícula de $\mathbf{0} 0.075 \mathrm{~mm}$.

7 Diferencias en porcentaje de oro recuperable equivalente a (3-5)/3.

8 Diferencias en porcentaje de oro recuperable equivalente a (4-6)/4.

A Para la zona A se aplica una constante de 0.92 (MLA) al $78 \%$ del Au que fue recuperado por gravimetría; y u $22 \%$ del oro que fue recuperado experimentalmente por cianuración a una molienda de $-0.84 \mathrm{~mm}$. Para la zon de 0.79 (MLA) al $84 \%$ del Au que fue recuperado por gravimetría; y una constante de 0.925 al $16 \%$ del oro que experimentalmente por cianuración a una molienda de $-0.84 \mathrm{~mm}$. Para la zona $\mathrm{C}$ se aplica una constante de 0 fue recuperado por gravimetría; y una constante de 0.90 al $11 \%$ del oro que fue recuperado experimentalmen molienda de $\mathbf{- 0 . 8 4} \mathrm{mm}$. Los datos experimentales son según pruebas de cianuración dinámica (en botellas).

B Para la zona A se aplica una constante de 0.92 (MLA) al $78 \%$ del Au que fue recuperado por gravimetría; y u $22 \%$ del oro que fue recuperado experimentalmente por cianuración a una molienda de $\mathbf{- 0 . 0 7 5} \mathrm{mm}$. Para la zo constante de 0.79 (MLA) al $84 \%$ del Au que fue recuperado por gravimetría; y una constante de 0.983 al $16 \%$ experimentalmente por cianuración a una molienda de - $0.075 \mathrm{~mm}$. Para la zona $\mathrm{C}$ se aplica una constante de que fue recuperado por gravimetría; y una constante de 0.913 al $11 \%$ del oro que fue recuperado experiment una molienda de $-0.075 \mathrm{~mm}$. Los datos experimentales son según pruebas de cianuración dinámica (en botellas). 


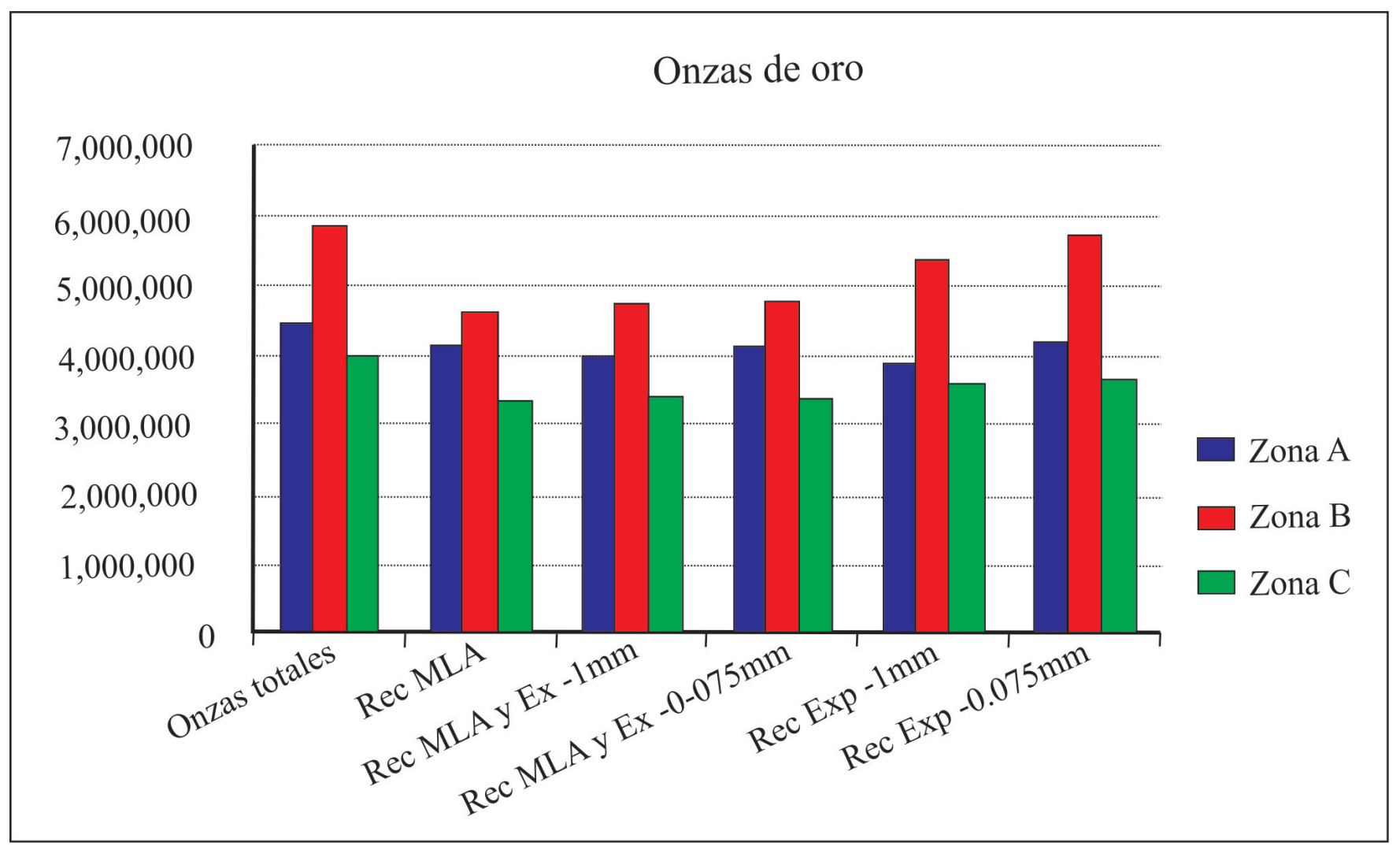

Figura 13 Onzas totales de oro y onzas recuperables utilizando los parámetros de la Tabla 6. Los grupos de columnas corresponden del 1 al 6 de la misma Tabla 6.

y las leyes ensayadas en los testigos usados para las pruebas de cianuración. Las leyes de cabeza analizadas para gravimetría dieron respectivamente: Zona A: $9.8 \mathrm{~g} / \mathrm{t}$ Au, Zona B: $2.5 \mathrm{~g} / \mathrm{t}$ Au y Zona $\mathrm{C}: 1.3 \mathrm{~g} / \mathrm{t} \mathrm{Au}$; mientras que las analizadas para cianuración dieron: Zona A: $15.5 \mathrm{~g} / \mathrm{t} \mathrm{Au}$, Zona B: $5.7 \mathrm{~g} / \mathrm{t}$ Au y Zona C: $1.5 \mathrm{~g} / \mathrm{t}$ Au. Hay también diferencias parecidas en las leyes de cabeza calculadas; casi siempre éstas son superiores a las analizadas, lo cual indicaría que siempre se recuperará más oro del ensayado. Las diferencias se deben seguramente a la presencia de oro grueso, característica muy conocida en La Herradura (Cossio et al., 2003).

Nuestros datos experimentales de concentraciones gravimétricas indican que se puede recuperar desde 78\% (Zona A) hasta un 84\% (Zona C), lo cual muestra que las recuperaciones actuales por este método pueden mejorarse a nivel industrial, puesto que en el circuito actual de la planta, entre septiembre de 2015 y febrero de 2016, se recuperó el $65.1 \%$ de oro en los concentradores Knelson instalados, antes de su paso a la cianuración dinámica. Dichos concentrados tuvieron leyes de 782 $\mathrm{g} / \mathrm{t}$ de $\mathrm{Au}$, con una cabeza calculada de $5.32 \mathrm{~g} / \mathrm{t}$ de Au.

Las principales asociaciones texturales de las partículas de oro identificadas con microscopía de reflexión y con MLA consisten en inclusiones en pirita y en esfalerita o en diversas gangas como cuarzo, albita, ortoclasa o ankerita, y asociaciones como películas en el borde de la pirita. La composición del oro es electrum con $74 \%$ de $\mathrm{Au}$ y 26\% de Ag y también se identificó la presencia de petzita $\left(\mathrm{Ag}_{3} \mathrm{AuTe}_{2}\right.$ y stutzita $(\mathrm{AgTe})$.

De la misma información de MLA se pudieron calcular constantes de recuperación para el oro de cada zona (Anexo 4 y Tabla 6) obteniéndose 
un total de onzas oro de 4,112,654 para la Zona A; 4,608,206 para la Zona B y 3,350,655 para la Zona C. Estos resultados son muy razonables cuando son comparados con la recuperación en pruebas de cianuración en botellas a la misma granulometría, consistentes en 3,889,140 para la zona A (-4\% de lo obtenido con MLA) y 3,589,988 para la Zona $\mathrm{C}(+6 \%$ de lo obtenido con MLA), aunque la diferencia es mayor para la Zona B: 5,366,519 (+13\% de lo obtenido con MLA). El cálculo de constantes de recuperación puede mejorarse cuando se combinan resultados de MLA y datos experimentales, aunque empeoran para la Zona B, como puede verse en la Tabla 6 . La diferencia en el oro recuperable de la Zona B se interpreta como la ausencia de una posible partícula de oro grueso existente pero no expuesta en las briquetas estudiadas. Esta diferencia define claramente el problema fundamental del oro en este tipo de depósitos, que es clásicamente un problema de muestreo y conminución de la muestra (Pitard, 1989a, 1989b; Cossio et al., 2003).

Lo anterior significa que es posible efectuar cálculos de recuperación confiables a partir de MLA y es posible observar también que no existe una diferencia significativa en recuperación utilizando un tamaño de partícula a $-0.84 \mathrm{~mm}$ (malla 20 ), en relación con la recuperación a un tamaño de partícula a $-0.075 \mathrm{~mm}$ (malla 200). Por ello, el proceso de remolienda que actualmente se realiza, antes de que el mineral pase a la planta de cianuración dinámica, puede ser innecesario, lo que generaría un ahorro importante en energía en éste, el parámetro más costoso en la minería (Bye, 2011), así como el principal responsable de emisiones de gases a la atmósfera (Bye, 2011).

Por otra parte, se considera que se puede tener una buena idea del oro recuperable a partir del oro identificado sobre las muestras in-situ. Por ejemplo, si consideramos las muestras de mineral de los barrenos estudiados para la Zona A (151 y 153) y si asumimos como oro no recuperable aquellas partículas inferiores a $30 \mu \mathrm{m}$ que corresponden a la mitad del tamaño de malla 200, resultaría como oro no recuperable el $9 \%$ y como oro recuperable el 91\%, casi el mismo de la constante calculada por MLA (92\%). Usando el mismo criterio sobre la muestra del barreno estudiado para la Zona B (146) y suponiendo que el oro no recuperable fuera aquel inferior a $20 \mu \mathrm{m}$, éste correspondería al $22 \%$ de oro no recuperable y el $78 \%$ a oro recuperable, que es prácticamente la misma constante calculada por MLA (79\%). Pudiéramos decir que una metodología más eficaz de trabajo puede ser estudiando simplemente los tramos más ricos en oro, en lugar de hacer compósitos por zonas, ya que en la conformación del compósito hay un procedimiento de dilución de oro y posteriormente otro de reconcentración por gravimetría; cada uno de estos procesos de dilución y concentración puede generar errores de muestreo y análisis.

Datos como los anteriores revaloran los datos sobre las características de formas, tamaños y texturas del oro que se identifican con mineralografía y la destacan como una técnica muy barata que puede aportar información muy valiosa sobre la economía de este tipo de yacimientos.

\section{Referencias}

Albinson, T.F., 1989, Vetas mesotermales auríferas del sector norte del Estado de Sonora, Memorias Técnicas, en XVIII Convención Nacional AIMMGM: Acapulco, Guerrero, México, Asociación de Ingenieros de Minas, Metalurgistas y Geólogos de México, 20-40.

Amstutz, G.C., 1961, Microscopy applied to mineral dressing: Quarterly of Colorado School of Mines, Colorado, 56, 445-481.

Anderson, T.H., Silver, L.T., 1979, The role of Mojave-Sonora Megashear in the tectonic evolution of northern Sonora, en Anderson, T.H., and Roldán-Quintana, J. (eds.), Geology of northern Sonora: Geological Society of America Field Trip Guidebook, San Diego, University of Pittsburgh, Universidad Nacional Autónoma de México, 59-68. 
Araux-Sánchez, E., 2000, Geología y yacimientos minerales de la Sierra Pinta, municipio de Puerto Peñasco, Sonora: Hermosillo, Sonora, Departamento de Geología, Universidad de Sonora, tesis de maestría, $121 \mathrm{p}$.

Birtel, S., Sandmann, D., Gutzmer, J., 2011, Geometallurgy: From ore to concentrate - a pilot study on the hydrothermal Au deposit at Cavanacaw, Northern Ireland: 11 bienanniel meeting SGA in Antofagasta, Chile, September 26-29. Mineralium Deposita, https://sga.conference-services.net/ resources/1054/2590/pdf/SGA2011_0028. pdf.

Bonnici, N.K., 2012, The mineralogical and textural characteristics of copper-gold deposits related to mineral processing attributes: Tasmania, Australia, University of Tasmania, tesis doctoral, $231 \mathrm{p}$.

Bye, A.R., 2011, Case studies demonstrating value from geometallurgy initiatives, in Proceedings of the International Geometallurgy Conference, The Australasian Institute of Mining and Metallurgy: Melbourne, Australia, 9-30.

Cossio, S., Noyola, J.L., González, P., Espinoza, R., 2003, Sampling and assaying of coarse gold at La Herradura-Minera Penmont S. de R.L., México, en Proceedings of the 35th Annual Meeting of the Canadian Mineral Processors, January 21-23: Ottawa, Canada, 249-262.

Dobby, G., Bennett, G., Bulled, D., Kosick, G., 2004, Geometallurgical modeling - The new approach to plant design and production forecasting/planning, and Mine/Mill Optimization, en Proceedings of 36th Annual Meeting of the Canadian Mineral Processors, January 20-22: Ottawa, Canada, Paper 15.

Durgin, D.C., Terán, P.I., 1996, La Choya Au deposit, NW Sonora, México, en Coyner, A.R., Fahey, P.L. (eds), Geology and ore deposits of the American Cordillera: Proceedings of the Geological Society of Nevada Symposium,: Reno/Sparks, Nevada, 1369-1373.

Fandrich, R., Gu, Y., Burrows, D., Moeller, K., 2007, Modern SEM-based mineral liberation analysis: International Journal of Mineral Processing, 84, 310-320.

Goldfarb, R.J., Groves, D.I., Gardoll, S., 2001, Orogenic gold and geologic time - a global synthesis: Ore Geology reviews, 18, 1-75.

Goldfarb, R.J., Hart, C.J., Marsh, E.E., 2008, Orogenic gold and evolution of the Cordilleran orogen, en Spencer, J.E., Titley, S.R. (eds), Ores and orogenesis: CircumPacific tectonics, geologic evolution, and ore deposits: Tucson, Arizona Geological Society Digest, 22, 311-323.

Goodall, W.R., Scales, P.J., 2007, An overview of the advantages and disadvantages of the determination of gold mineralogy by automated mineralogy: Minerals Engineering, 20, 506-517.

Gottlieb, P., Wilkie, D., Sutherland, D., HoTun, E., Suthers, S., Perera, K., Jenkins, B., Spencer, A., Butcher, A., 2000, Using quantitative electron microscopy for process mineralogy applications: JOM- The Journal of The Minerals, Metals \& Materials Society (TMS), 52, 24-25.

Groves, D.I., Goldfarb, R.J., Gebre-Mariam, M., Hagemann, S.G., Robert, F., 1998, Orogenic gold deposits: A proposed classification in the context of their crustal distribution and relationship to other geologic deposits types: Ore Geology Reviews, 13, 7-27.

Gu, Y., 2003., Automated scanning electron microscope based mineral liberation Analysis - an introduction to JKMRG/FEI Mineral Liberation Analizer: Journal of Mineral \& Materials Characterization \& Engineering, 2, 33-41. 
Hagni, R.D., 1978, Ore microscopy applied to beneficiation: Mining Engineering, 30, 1137-1147.

Hall, J.S., 1977, Composite mineral particles analysis by automated scanning electron microscopy. Julius Kruttschnitt Mineral Research Centre (JMRG): Brisbane, Australia, University of Queensland, tesis doctoral, $409 \mathrm{p}$.

Hallewell, M., 2009, Geometallurgy for mine data: Materials World, 17, 48-50.

Hartner, R., 2012, Integration and analysis of optical and MLA-based microscopy for optimisation of geometallurgical modelling and ore deposit characterisation: Brisbane, Australia, University of Queensland, tesis doctoral, $217 \mathrm{p}$.

Hoal, K.O., Stammer, J.G., Appleby, J., Botha, J., Ross, J.K., Botha, P.W., 2009, Research in quantitative mineralogy: Examples from diverse applications: Minerals Engineering, 22, 402-408.

Hunt, J., Berry, R., Bradshaw, D., 2011, Characterising chalcopyrite liberation and flotation potential: Examples from an IOCG deposit: Minerals Engineering, 24, 1271-1276.

Iriondo, A., Martínez-Torres, L.M., Kunk, M.J., Atkinson, W.W., Premo, W.R., McIntosh, W.C., 2005, Northward Laramide thrusting in the Quitovac region, northwestern Sonora, México: Implication for the juxtaposition of Paleoproterozoic basement blocks and the Mojave-Sonora megashear hypothesis, en Anderson, T.H., Nourse, J.A., McKee, J.W., Steier, M.B. (eds.), The Mojave-Sonora megashear hypothesis: Development, assessment, and alternatives: Boulder, Colorado, Geological Society of America Special Paper, 393, 631-669.

Izaguirre, A., 2009, El basamento Paleoproterozoico ( 1.71-1.68 Ga) Yavapai en el área Mina La Herradura en el NW de Sonora: Sus implicaciones para el desarrollo del margen magmático continental Mesozoico-Cenozoico del NW de México: Querétaro, Centro de Geociencias, Universidad Nacional Autónoma de México, tesis de maestría, $201 \mathrm{p}$.

Izaguirre, A., Iriondo, A., Caballero-Martínez, J.A., Moreira-Rivera, F., Espinosa-Arámburu, E., 2012, Homogeneidad geoquímica de la alteración hidrotermal del cinturón de oro orogénico del NW de Sonora, México: Estudio de balance de masas en rocas encajonantes de la mineralización: Boletín de la Sociedad Geológica Mexicana, 64, 119-153.

Izaguirre, A., Camprubi, A., Iriondo, A., 2017, Mesozoic orogenic gold deposits in México: Ore Geology Reviews, 81, 1172-1183.

Keeney, L., 2010, The development of a novel method for integrating geometallurgical mapping and orebody modelling: Brisbane, Australia, Sustainable Minerals Institute, The University of Queensland, tesis doctoral, 309 p.

Lamberg, P., 2011, Particles - the bridge between geology and metallurgy, en Alatalo, J. (ed.), Conference in Minerals Engineering: Lulea, Lulea University of Technology, 1-16.

Leichliter, S.E., 2013, Gold deportment and geometallurgical recovery model for the La Colosa, Porphyry Gold Deposit, Colombia: Tasmania, Australia, University of Tasmania, tesis doctoral, $176 \mathrm{p}$.

Leichliter, S.E., Hunt, J., Berry, R., Keeney, L., Montoya, P., Chamberlain, V., Jahoda, R., Drews, U., 2011, Development of a predictive geometallurgical recovery model for the La Colosa, Porphyry Gold Deposit, Colombia, in Proceedings of the First AusIMM International Geometallurgy Conference, Melbourne, 85-92. 
Marsden, J., House, I., 1992, The chemistry of gold extraction: Londres, Ellis Horwood, 597 p.

Meza-Figueroa, D.M., 1992, Estudio metalogenético del yacimiento de oro de Tajitos: Hermosillo, Sonora, Departamento de Geología, Universidad de Sonora, tesis de licenciatura, $112 \mathrm{p}$.

Miller, P.R., Reid, A.F., Zuiderwyk, M.A., 1982, QEM*SEM image analysis in the determination of modal assays, mineral associations and mineral liberation, in XIV International Processing Congress, Toronto, Canada: CIM-VIII-3.1-VIII-3.20.

Noriega-Martínez, O., 2006, Evidencias de dos etapas de mineralización de oro mesotermal en las áreas de Tajitos, Juárez-San Francisco en la región de Caborca, Sonora, México: Hermosillo, Sonora, Departamento de Geología, Universidad de Sonora, tesis de maestría, $85 \mathrm{p}$.

Ochoa-Landín, L., Pérez-Segura, E., Del RíoSalas, R., Valencia-Moreno, M., 2011, Depósitos minerales de Sonora, México, en Calmus, T. (ed.), Panorama de la geología de Sonora: Boletín del Instituto de Geología, 118, 1-33.

Ornelas-Macías, A., Verdugo-Noriega, G., 2009, Caracterización geológica, estructural y de mineralización en el área de Karina, municipio de Caborca, Sonora, México: Hermosillo, Sonora, Departamento de Geología, Universidad de Sonora, tesis de licenciatura, $110 \mathrm{p}$.

Pérez-Barnuevo, L., Castroviejo, R., Berrezueta, E., 2009, Análisis digital de imágenes RGB aplicado a la geometalurgia. Puesta a punto del equipo y su potencial frente a métodos tradicionales: Macla, Revista de la Sociedad Española de Mineralogía, 11, 143-144.

Pérez Segura, E., 2003, Contribuciones de la mineragrafía a los procesos industriales de recuperación de minerales: México, Conferencia de ingreso a la Academia de Ingeniería (AI), http://www.ai.org. $\mathrm{mx} /$ presentacion/contribuciones-de-lamineragrafia-los-procesos-industriales-derecuperación-de-minerales, $28 \mathrm{p}$.

Pérez Segura, E., 1989. Descubrimiento de una paragénesis de $\mathrm{Au}-\mathrm{Te}$ en el yacimiento de San Francisco, Sonora. Memorias Técnicas, en XVIII Convención Nacional AIMMGM: Acapulco, Guerrero, México, Asociación de Ingenieros de Minas, Metalurgistas y Geólogos de México, 63 - 79 .

Pérez Segura, E., Cheilletz, A., HerreraUrbina, S., Hanes, Y.J., 1996, Geología, mineralización, alteración hidrotermal y edad del yacimiento de oro de San Francisco - Un depósito mesotermal en el Noroeste de México: Revista Mexicana de Ciencias Geológicas, 13, 65-89.

Pérez Segura, E., Gaspar, J.C., Brown, F., 2013, La geometalurgia: una excelente herramienta multidisciplinaria para la vinculación universidad - industria: Revista Epistemus, 14, 73-76.

Petruk, W., 2000, Applied mineralogy in the mining industry: Amsterdam, Elsevier, 268 p.

Pitard, F.F., 1989a, Pierre Gy's sampling theory and sampling practice, Vol. I. Heterogeneity and sampling: Boca Raton, CRG Press, 214 p.

Pitard, F.F.,1989b, Pierre Gy's sampling theory and sampling practice, Vol. II. Sampling correctness and sampling practice: Boca Raton, CRG Press, 247 p.

Poole, F., Sandberg, C.A., Amaya-Martínez, R., 2004, Biostratigraphy and paleotectonic setting of Paleozoic rocks north of Estacion Sahuaro, Northwestern Sonora, Mexico: Denver, Colorado, Geological Society of America Denver Annual Meeting, Abstracts with Programs, 36, 5, p.99. 
Quintanar-Ruiz, FJ., 2008, La Herradura ore deposit: An orogenic gold deposit in northwestern Mexico: Tucson, Arizona, University of Arizona, tesis de maestría, 97 p.

Ramdohr, P., 1980, The ore minerals and their intergrowths: Berlin, Pergamon Press, 2 v., $1207 \mathrm{p}$.

Reid, A.F., Gottlieb, P., MacDonald, J.K., Miller, P.R., 1984, QEM*SEM image analysis of ore minerals: volume fraction, liberation and observational variances, in Applied Mineralogy. Proceedings of the Second International Congress on Applied Mineralogy in the Minerals Industry: Los Angeles, The Metallurgical Society of AIME, 191-204.

Romero-Valle, J.M., 2005, Geología y métodos de exploración de la mina La herradura, municipio de Caborca: Hermosillo, Sonora, Departamento de Geología, Universidad de Sonora, tesis de licenciatura, $55 \mathrm{p}$.

Romero-Valle, J.M., 2017, Ensayo de un modelo geometalúrgico para la zona de sulfuros primarios profundos de la mina La Herradura en Sonora, México: Hermosillo, Sonora,
Departamento de Geología, Universidad de Sonora, tesis de maestría, $67 \mathrm{p}$.

Silberman, M.L., Giles, D.A., Graubard, C., 1988, Characteristics of gold deposits in Northern Sonora, Mexico: A preliminary report: Economic Geology, 83, 1966-1974.

Thoms, J.A., 1998, Exploration and geology of the Choya Sur gold deposit, Sonora, Mexico, en Clark, K.F. (ed.), Gold deposits of northern Sonora, Mexico: Society of Economic Geologists Guidebook, 30, 157-176.

Torre, J.C. de la, 2009, Geología del yacimiento mina La herradura, en Clark, K.F., SalasPizá, G., Cubillas-Estrada, R. (eds.), Geología Económica de México, 2a. Ed: México, Servicio Geológico Mexicano - Asociación de Ingenieros de Minas, Metalurgistas y Geólogos de México, p. 844-851.

Torre, J.C. de la, 2010, Paragenetic investigation of the hydrothermal alterations and gold mineralization of Centauro and Valles at La Herradura gold district in Sonora, Mexico: Kingston, Ontario, Canada, Queen's University, tesis de maestría, $55 \mathrm{p}$

Zhou, J., Gu, Y., 2016, Geometallurgical characterization and automated mineralogy of gold ores: Gold Ore Processing, 95-111. 


\section{Anexo 1}

Bases que sustentan la geometalurgia según PérezSegura et al. (2013).

- La determinación y control de procesos basada sólo en una química analítica general puede ser engañosa.

- Los depósitos minerales están formados por más de un tipo de mena.

- Los minerales de ganga son mucho más abundantes que los de mena. Estos minerales pueden determinar, por ellos mismos y por características estructurales del depósito, condiciones de procesos de minado, quebrado, molienda, flotación o cianuración, su confinamiento ambiental sostenible y su estudio para posibles reutilizaciones futuras.

- En algunas menas el metal importante ocurre en más de un mineral y cada mineral tiende a presentar diferente comportamiento durante los procesos, por lo que las recuperaciones de un elemento pueden ser diferentes para diferentes minerales. Por otra parte, es el tipo de mineral de mena recuperado el que determina, por ejemplo, la ley de un concentrado obtenido por cualquier proceso.

\section{Anexo 2}

Descripción de la preparación de compósitos y toma de muestras.

- Se seleccionaron barrenos y tramos representativos de mineralización en sulfuros. Se escogieron tramos de leyes superiores a las medias para tener mayor facilidad de observación de las características del oro.

- De cada barreno y de cada tramo (2 metros por tramo), se extrajeron 200 gramos de material triturado representativo utilizando un muestreador rotatorio.

- De cada barreno seleccionado se hizo un compósito.
- De cada compósito por barreno se extrajeron 3 muestras iguales de 60 gramos cada una en muestreador rotatorio. Estas 3 muestras se utilizaron así: una para análisis de $\mathrm{Au}$ al fuego, otra para análisis de Au soluble en cianuro de sodio $(\mathrm{CNNa})$ y otra para análisis de elementos mayores y trazas por Plasma Inductivamente Acoplado (ICP).

- Con todos los compósitos por barreno se hizo un solo compósito por zona (uno para la Zona A, otro para la B y otro para la G). Cada uno de estos compósitos pesó aproximadamente $15 \mathrm{~kg}$.

- De cada compósito por zona se extrajeron 3 muestras iguales de 60 gramos, cada una en muestreador rotatorio. Estas 3 muestras se utilizaron: una para análisis de Au al fuego, otra para análisis de Au soluble en CNNa y otra para análisis por elementos mayores y trazas por ICP.

- Las muestras de compósito por zona (3) se molieron a un tamaño de $-0.84 \mathrm{~mm}$ (malla -20).

- Después de la extracción de muestras para análisis, cada compósito molido se pasó por un concentrador gravimétrico de tipo Knelson. Al final se obtuvo de cada muestra un concentrado gravimétrico y una cola (total 3 concentrados y 3 colas).

- Los concentrados y las colas se analizaron por Au total al fuego.

- De cada concentrado y de cada cola se hicieron secciones pulidas para ser estudiadas al microscopio mineralográfico y al microscopio electrónico de barrido.

\section{Anexo 3}

Premisas básicas para la realización del modelo geometalúrgico:

- La molienda utilizada para la realización de este trabajo fue de $-0.84 \mathrm{~mm}$ (malla 20), tamaño a partir del cual se efectuaron los 
concentrados gravimétricos de los cuales se hicieron las briquetas estudiadas con Mineral Liberation Analizer (MLA).

- Se hicieron pruebas experimentales de cianuración dinámica en botellas de muestras testigo, al mismo tamaño de partícula de $-0.84 \mathrm{~mm}$.

- Se hicieron también pruebas experimentales de cianuración dinámica en botellas de las muestras testigo a un tamaño de partícula de $-0.075 \mathrm{~mm}$ (malla 200).

Las leyes de oro analizadas para conocer contenidos de oro y calcular recuperaciones fueron a partir de análisis al fuego, sin embargo, no hay que olvidar que la planta de lixiviación dinámica industrial opera con cianuración, por lo que la recuperación es a partir de oro soluble en CNNa. El tamaño de la partícula de la planta es de $-0.103 \mathrm{~mm}$ (malla 150), aunque fue diseñada para un tamaño de partícula de $-0.075 \mathrm{~mm}$ (malla 200).

\section{Anexo 4}

Cálculos de oro recuperado utilizando datos de MLA y utilizando datos experimentales de cianuración dinámica.

Contenido de Mineral y metal Zona A - Constante de recuperación utilizada: 92\%

\begin{tabular}{|l|c|c|c|c|c|}
\multicolumn{5}{|c|}{ Contenido de Mineral y metal Zona A - Constante de recuperación utilizada: 92\% } \\
\hline \multicolumn{1}{|c|}{ Cut Off } & Ton & Ley & Cont. Gr & Onzas & Oz Rec. \\
\hline Total bloques & $1,456,850,227$ & 0.095 & $139,041,151$ & $4,470,276$ & $4,112,654$ \\
\hline$>0.3$ & $136,210,969$ & 0.895 & $121,862,756$ & $3,917,978$ & $3,604,540$ \\
\hline$>1.5$ & $17,926,124$ & 2.298 & $41,195,458$ & $1,324,465$ & $1,218,507$ \\
\hline 3.0 & $1,993,484$ & 5.007 & $9,980,850$ & 320,892 & 295,220 \\
\hline
\end{tabular}

Contenido de Mineral y metal Zona B - Constante de recuperación utilizada: 79\%

\begin{tabular}{|l|l|l|l|r|r|}
\hline \multicolumn{1}{c|}{ Cut Off } & \multicolumn{1}{c|}{ Ton } & \multicolumn{1}{c|}{ Ley } & \multicolumn{1}{c}{ Cont. Gr } & Onzas & \multicolumn{1}{c}{ Oz Rec. } \\
\hline Total bloques & $3,623,551,669$ & 0.05 & $181,431,964$ & $5,833,173$ & $4,608,206$ \\
$>0.3$ & $196,542,674$ & 0.777 & $152,808,661$ & $4,912,912$ & $3,881,200$ \\
$>1.5$ & $17,549,734$ & 2.177 & $38,209,754$ & $1,228,472$ & 970,493 \\
$>3.0$ & $1,733,347$ & 4.214 & $7,303,768$ & 234,822 & 185,509
\end{tabular}

Contenido de Mineral y metal Zona C - Constante de recuperación utilizada: 84\%

\begin{tabular}{l|l|l|l|l|l|}
\hline \multicolumn{1}{c|}{ Cut Off } & \multicolumn{1}{c|}{ Ton } & \multicolumn{1}{c|}{ Ley } & \multicolumn{1}{c}{ Cont. Gr } & \multicolumn{1}{c}{ Onzas } & \multicolumn{1}{c}{ Oz Rec. } \\
\hline Total bloques & $3,889,041,478$ & 0.032 & $124,067,902$ & $3,988,875$ & $3,350,655$ \\
$>0.3$ & $148,297,603$ & 0.597 & $88,491,360$ & $2,845,063$ & $2,389,853$ \\
$>1.5$ & $3,981,510$ & 1.81 & $7,204,969$ & 231,645 & 194,582 \\
$>3.0$ & 18,000 & 3.166 & 56,988 & 1,832 & 1,539
\end{tabular}

Cálculos con datos de recuperación de MLA sobre los concetrados obtenidos por gravimetría a una granulometría de $-0.84 \mathrm{~mm}$ (-20 mallas). 


\begin{tabular}{|c|c|c|c|c|c|}
\multicolumn{5}{|c|}{ Contenido de Mineral y metal Zona A - Constante de recuperación utilizada 87\% } \\
\hline Cut Off & Ton & Ley & Cont. Gr & Onzas & Oz Rec. \\
\hline All blocks & $1,456,850,227$ & 0.095 & $139,041,151$ & $4,470,276$ & $3,889,140$ \\
\hline$>0.3$ & $136,210,969$ & 0.895 & $121,862,756$ & $3,917,978$ & $3,408,641$ \\
\hline$>1.5$ & $17,926,124$ & 2.298 & $41,195,458$ & $1,324,465$ & $1,152,284$ \\
\hline$>3.0$ & $1,993,484$ & 5.007 & $9,980,850$ & 320,892 & 279,176 \\
\hline
\end{tabular}

\begin{tabular}{|l|l|l|r|r|r|}
\multicolumn{5}{c|}{ Contenido de Mineral y metal Zona B - Constante de recuperación utilizada 92\% } \\
\hline \multicolumn{1}{|c|}{ Cut Off } & \multicolumn{1}{c|}{ Ton } & \multicolumn{1}{c|}{ Ley } & Cont. Gr & Onzas & Oz Rec. \\
\hline All blocks & $3,623,551,669$ & 0.05 & $181,431,964$ & $5,833,173$ & $5,366,519$ \\
$>0.3$ & $196,542,674$ & 0.777 & $152,808,661$ & $4,912,912$ & $4,519,879$ \\
$>1.5$ & $17,549,734$ & 2.177 & $38,209,754$ & $1,228,472$ & $1,130,194$ \\
$>3.0$ & $1,733,347$ & 4.214 & $7,303,768$ & 234,822 & 216,036 \\
\hline
\end{tabular}

\begin{tabular}{|l|l|l|l|r|r|}
\multicolumn{5}{|c|}{ Contenido de Mineral y metal Zona C - Constante de recuperación utilizada 90\% } \\
\hline \multicolumn{1}{c|}{ Cut Off } & \multicolumn{1}{c}{ Ton } & \multicolumn{1}{c}{ Ley } & \multicolumn{1}{c|}{ Cont. Gr } & Onzas & Oz Rec. \\
\hline All blocks & $3,889,041,478$ & 0.032 & $124,067,902$ & $3,988,875$ & $3,589,988$ \\
\hline$>0.3$ & $148,297,603$ & 0.597 & $88,491,360$ & $2,845,063$ & $2,560,557$ \\
$>1.5$ & $3,981,510$ & 1.81 & $7,204,969$ & 231,645 & 208,481 \\
$>3.0$ & 18,000 & 3.166 & 56,988 & 1,832 & 1,649
\end{tabular}

Cálculos con datos de recuperación a partir de pruebas de cianuración en botella a una granulometría de $-0.84 \mathrm{~mm}$ (-20 mallas).

\begin{tabular}{|c|c|c|c|c|c|}
\multicolumn{5}{|c|}{ Contenido de Mineral y metal Zona A - Constante de recuperación utilizada 94\% } \\
\hline \multicolumn{1}{|c|}{ Cut Off } & Ton & Ley & Cont. Gr & Onzas & Oz Rec. \\
\hline All blocks & $1,456,850,227$ & 0.095 & $139,041,151$ & $4,470,276$ & $4,202,060$ \\
\hline$>0.3$ & $136,210,969$ & 0.895 & $121,862,756$ & $3,917,978$ & $3,682,899$ \\
\hline$>1.5$ & $17,926,124$ & 2.298 & $41,195,458$ & $1,324,465$ & $1,244,997$ \\
\hline 3.0 & $1,993,484$ & 5.007 & $9,980,850$ & 320,892 & 301,638 \\
\hline
\end{tabular}

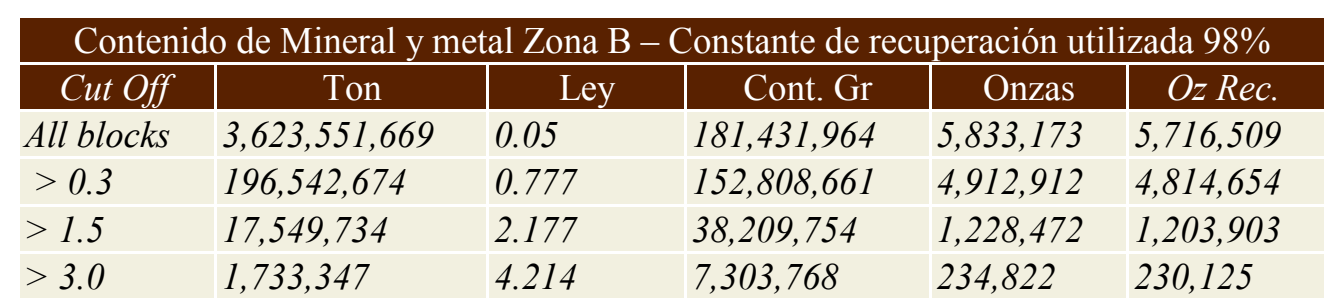

\begin{tabular}{|c|l|l|l|l|l|}
\multicolumn{5}{|c|}{ Contenido de Mineral y metal Zona C - Constante de recuperación utilizada 91\% } \\
\hline \multicolumn{1}{c|}{ Cut Off } & \multicolumn{1}{c|}{ Ton } & \multicolumn{1}{c}{ Ley } & \multicolumn{1}{c|}{ Cont. Gr } & \multicolumn{1}{c|}{ Onzas } & \multicolumn{1}{c|}{ Oz Rec. } \\
\hline All blocks & $3,889,041,478$ & 0.032 & $124,067,902$ & $3,988,875$ & $3,629,876$ \\
$>0.3$ & $148,297,603$ & 0.597 & $88,491,360$ & $2,845,063$ & $2,589,007$ \\
$>1.5$ & $3,981,510$ & 1.81 & $7,204,969$ & 231,645 & 210,797 \\
$>3.0$ & 18,000 & 3.166 & 56,988 & 1,832 & 1,667
\end{tabular}

Cálculos con datos de recuperación a partir de pruebas de cianuración en botella a una granulometría de $-0.075 \mathrm{~mm}(-200$ mallas $)$. 